\title{
Signaling pathways predisposing to chronic kidney disease progression
}

\author{
Mohamad Zaidan, ${ }^{1,2}$ Martine Burtin, ${ }^{1}$ Jitao David Zhang, ${ }^{3}$ Thomas Blanc, ${ }^{1,4}$ Pauline Barre, ${ }^{1}$ \\ Serge Garbay, ${ }^{1}$ Clément Nguyen, ${ }^{1}$ Florence Vasseur, ${ }^{1}$ Lucie Yammine, ${ }^{1}$ Serena Germano, ${ }^{1}$ Laura Badi, ${ }^{3}$ \\ Marie-Claire Gubler, ${ }^{5}$ Morgan Gallazzini, ${ }^{1}$ Gérard Friedlander, ${ }^{1,6}$ Marco Pontoglio, ${ }^{1}$ and Fabiola Terzi ${ }^{1}$ \\ 'Institut National de la Santé et de la Recherche Médicale (INSERM), U1151, CNRS UMR 8253, Institut Necker Enfants \\ Malades (INEM), Department of Growth and Signaling, Université de Paris, Paris, France. ²Service de Néphrologie- \\ Transplantation, Hôpital Bicêtre, Assistance Publique - Hôpitaux de Paris (AP-HP), Le Kremlin-Bicêtre, France. \\ ${ }^{3}$ Pharmaceutical Sciences, Roche Pharmaceutical Research and Early Development, Roche Innovation Center Basel, \\ Basel, Switzerland. ${ }^{4}$ Service de Chirurgie Viscérale et Urologie Pédiatrique, Hôpital Necker Enfants Malades, AP-HP, Paris, \\ France. ${ }^{5}$ INSERM U1163, Institut Imagine, Université de Paris, Paris, France. ${ }^{6}$ Service d'Explorations Fonctionnelles, Hôpital \\ Européen Georges Pompidou, AP-HP, Paris, France.
}

The loss of functional nephrons after kidney injury triggers the compensatory growth of the remaining ones to allow functional adaptation. However, in some cases, these compensatory events activate signaling pathways that lead to pathological alterations and chronic kidney disease. Little is known about the identity of these pathways and how they lead to the development of renal lesions. Here, we combined mouse strains that differently react to nephron reduction with molecular and temporal genome-wide transcriptome studies to elucidate the molecular mechanisms involved in these events. We demonstrated that nephron reduction led to 2 waves of cell proliferation: the first one occurred during the compensatory growth regardless of the genetic background, whereas the second one occurred, after a quiescent phase, exclusively in the sensitive strain and accompanied the development of renal lesions. Similarly, clustering by coinertia analysis revealed the existence of $\mathbf{2}$ waves of gene expression. Interestingly, we identified type I interferon (IFN) response as an early (first-wave) and specific signature of the sensitive (FVB/N) mice. Activation of type I IFN response was associated with $\mathrm{G}_{1} / \mathrm{S}$ cell cycle arrest, which correlated with p21 nuclear translocation. Remarkably, the transient induction of type I IFN response by poly(I:C) injections during the compensatory growth resulted in renal lesions in otherwise-resistant C57BL6 mice. Collectively, these results suggest that the early molecular and cellular events occurring after nephron reduction determine the risk of developing late renal lesions and point to type I IFN response as a crucial event of the deterioration process.

Conflict of interest: The authors have declared that no conflict of interest exists.

Copyright: ( 2020 , American Society for Clinical Investigation.

Submitted: November 13, 2018

Accepted: April 8, 2020

Published: May 7, 2020.

Reference information: /CI Insight. 2020;5(9):e126183.

https://doi.org/10.1172/jci.

insight.126183.

\section{Introduction}

Chronic kidney disease (CKD), one of the major public health challenges of the 21st century, is characterized by the progressive decline of renal function to end-stage renal disease (ESRD) that may occur, irrespective of the cause of the renal damage, once a critical number of nephrons has been lost (1-3). Approximately $10 \%$ to $15 \%$ of adults suffer from CKD, and the incidence of ESRD increases by $6 \%$ to $8 \%$ per year $(1,2)$. Despite efforts by the health care community, the survival and quality of life of patients with ESRD remain poor due in large part to the deleterious sequelae. Because of the persistently poor outcome of ESRD, current clinical research efforts focus on preventive strategies to slow down the rate of CKD progression.

The mechanisms of CKD progression are poorly understood. Attempts to dissect the molecular basis of $\mathrm{CKD}$ have been facilitated by the development of several experimental animal models. Among these, the subtotal nephrectomy model is a mainstay because this model recapitulates many features of human $\mathrm{CKD}$, including hypertension, proteinuria, and glomerular and tubulointerstitial lesions (4-6). Over the last 50 years, the study of this model has led to the discovery of critical pathways and, more importantly, to the design of therapeutic strategies to delay CKD progression, such as renin-angiotensin inhibitors $(7,8)$.

It has been shown that the reduction of the number of functional nephrons triggers molecular and cellular events, promoting compensatory growth of the remaining ones to maintain kidney function (4-6). 
In some cases, these compensatory events may activate pathways that lead to pathological changes marked by the development of glomerular sclerosis, tubular atrophy and cysts, interstitial fibrosis, and mononuclear cell infiltration $(9,10)$. The identity of the cellular pathways engaged during the renal compensatory growth process and how they lead to the development of later renal lesions are still not known.

Epidemiological studies indicate that the rate of CKD progression can vary significantly among individuals. Although clinical observations have disclosed the importance of environmental factors in the biological processes leading to renal deterioration, several studies have highlighted the critical role played by genetic factors $(1,2)$. Similarly, experimental models of CKD have shown that the development of renal lesions is genetically determined in mice $(11,12)$. By applying subtotal nephrectomy to 6 mouse strains, we previously demonstrated that only 1 , the $\mathrm{FVB} / \mathrm{N}$, develops severe renal lesions after nephron reduction, whereas the others undergo compensatory growth alone $(9,13,14)$. The development of renal lesions in $\mathrm{FVB} / \mathrm{N}$ mice is associated with an increase of cell proliferation. Notably, previous studies demonstrated that the pharmacological or molecular inhibition of cell proliferation attenuates, to some extent, the progression of renal lesions in several experimental models of $\mathrm{CKD}$, indicating that cell proliferation is crucially involved in the deterioration process. More recently, it has been proposed that a cell cycle $\mathrm{G}_{2} / \mathrm{M}$ arrest may play a critical role in lesion development during CKD (15). Indeed, this arrest favors the expression of profibrotic genes. Nevertheless, tubular cell proliferation is also required for the renal compensatory growth that follows nephron reduction. Whether the same genetic networks trigger cell proliferation during the compensatory and deterioration phases is still unknown.

Here, we took advantage of 2 strains of mice, FVB/N and C57BL6, that differently react to nephron reduction to dissect the genetic networks that underlie renal compensation and deterioration. Using unbiased temporal profiling of whole-kidney transcriptome, we identified type I interferon (IFN) response as a specific signature of the sensitive $\mathrm{FVB} / \mathrm{N}$ mice during the first peak of cell proliferation. This signature was associated with a $\mathrm{G}_{1} / \mathrm{S}$ cell cycle arrest, which correlated with p21 nuclear translocation. Finally, we demonstrated that the early induction of type I IFN response led to glomerular lesions in initially resistant C57BL6 mice, suggesting that this pathway might be the critical link between the renal compensation and deterioration processes.

\section{Results}

Tubular cell proliferation characterizes both compensatory growth and renal deterioration processes but in a strain-dependent manner. To better understand the nature of the events that lead to either the renal compensatory growth or the lesion development after nephron reduction (via subtotal nephrectomy, Nx), we first studied the temporal modifications of renal architecture and the pattern of tubular cell proliferation in the sensitive FVB/N (hereafter named FVB) and resistant C57BL6 (hereafter named B6) mice at 2, 28, and 56 days after Nx. Histological analysis showed acute tubular damage in both FVB and B6 mice 2 days after Nx (Figure 1A and Supplemental Figure 1A; supplemental material available online with this article; https://doi.org/10.1172/jci.insight.126183DS1). Tubular lesions, which were particularly severe in the scar regions, were similar between the 2 strains of mice (Supplemental Figure 1A). Consistently, the expression of LCN2, a marker of kidney injury, increased in remnant kidneys of both FVB and B6 mice (Supplemental Figure 1B). Ki-67 staining showed a first robust burst of tubular cell proliferation 2 days after Nx regardless of the genetic background (Figure 1B and Supplemental Figure 1C). This was associated with a progressive increase of the kidney-to-body weight ratio, suggesting that proliferation thrusts the compensatory growth of remaining nephrons (Figure 1C and Supplemental Figure 2). Of note is the observation that compared with the estimated remnant kidney weight left at the time of surgery, kidney weight at day 2 increased about 30\%. At day 28, apart from the surgical scars, the renal parenchyma displayed almost normal morphology regardless of the genetic background (Figure 1A). Cell proliferation was also dramatically switched off and became almost undetectable (Figure 1B and Supplemental Figure 1C). Remarkably, after this "quiescent phase," we observed a sudden second wave of cell proliferation but exclusively in the FVB mice. This was associated with the development of chronic renal lesions, i.e., glomerular sclerosis, tubular dilations, and interstitial fibrosis and inflammation (Figure 1A and Supplemental Figure 3). A careful time course quantification of renal lesions showed that the severity of renal lesions was dramatically increased in all nephron compartments at day 56, mainly in NxFVB mice (Supplemental Figure 3). This "deterioration phase" was characterized by a further increase of the kidney-to-body weight ratio in FVB mice (Figure 1C and Supplemental Figure 2). 
A

ShB6

ShFVB
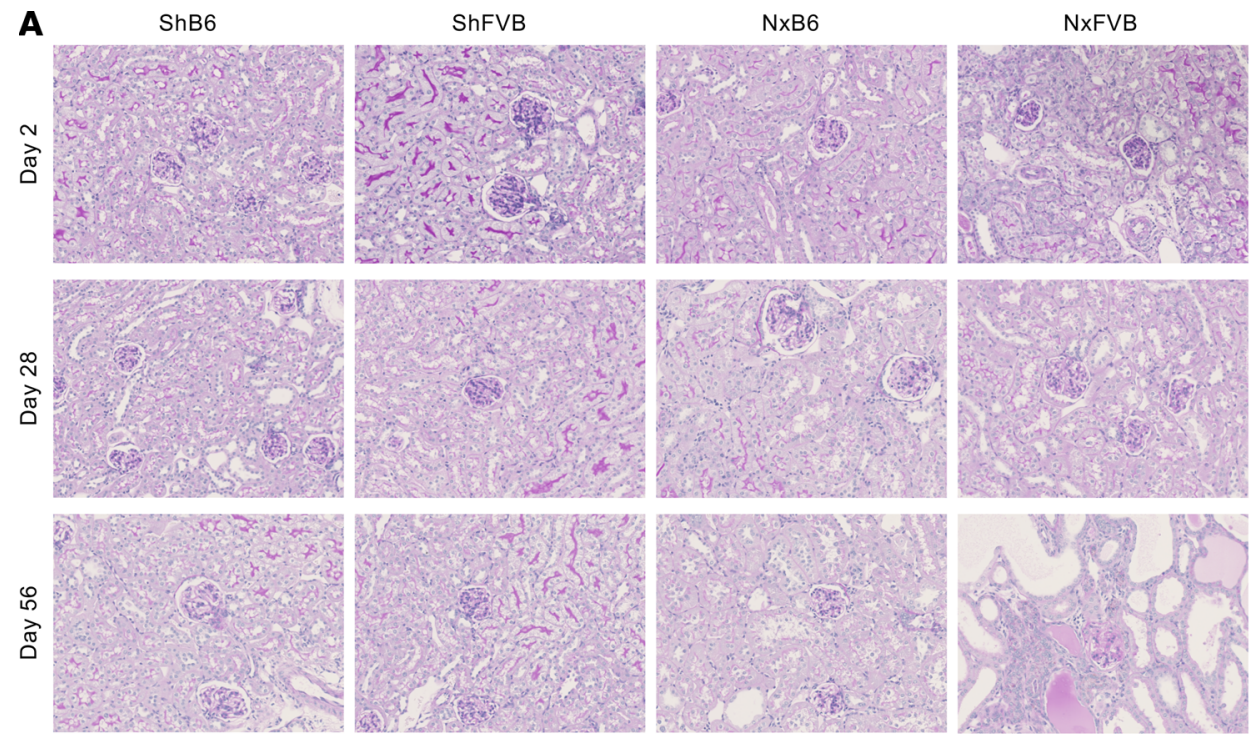

B

Day 2

Day 28

Day 56
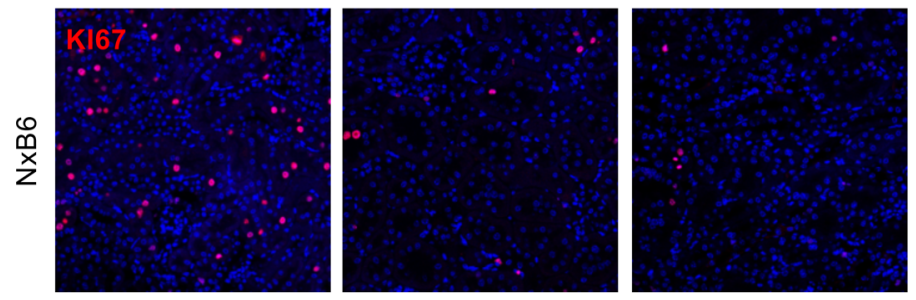

Day 2

Day 28

Day 56
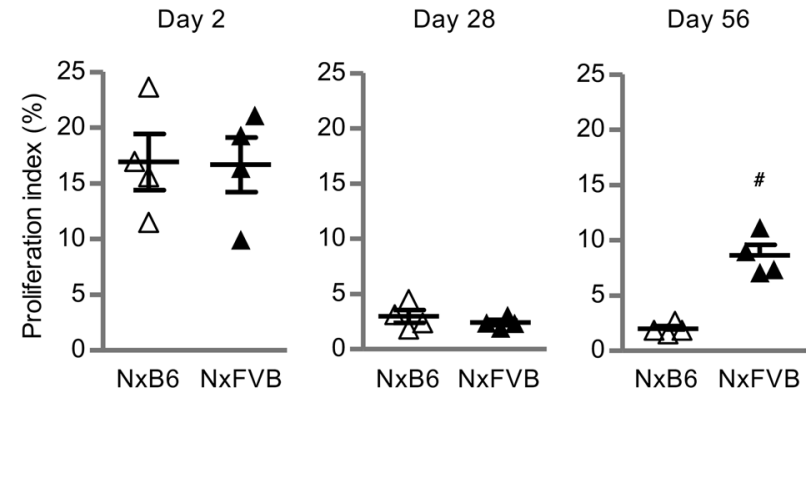

$\underset{\substack{l \\ \text { 妾 }}}{m}$
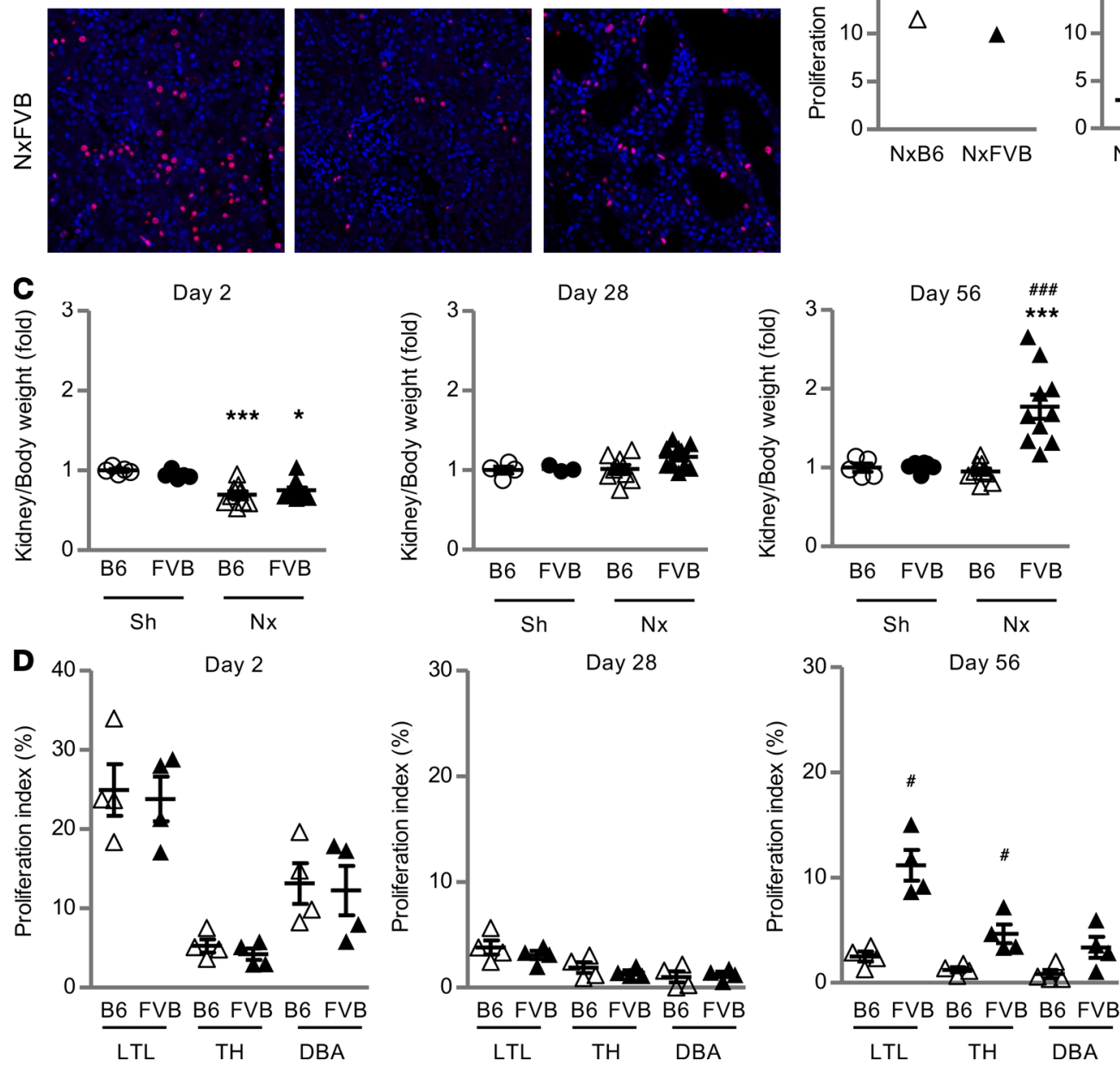
Figure 1. Nephron reduction leads to 2 waves of cell proliferation in sensitive FVB mice. (A) Representative cortical sections from FVB and B6 mice 2,28 , and 56 days after sham operation (Sh) or subtotal nephrectomy $(\mathrm{Nx})$ stained with periodic acid-Schiff (original magnification, $\times 200 ; n=4-6$ and 10-12 for Sh and Nx, respectively, in each strain at each time point). (B) Representative images of Ki-67 immunostaining in B6 and FVB mice 2 , 28, and 56 days after $\mathrm{Nx}$ and quantification of tubular cell proliferation index (original magnification, $\times 400 ; n=4 \mathrm{Nx}$ mice at least in each strain at each time point). Data are shown as mean \pm SEM. Mann-Whitney $U$ test. NxFVB versus NxB6 mice: ${ }^{\#}<0.05$. (C) Time course analysis of kidneyto-body weight ratio in B6 and FVB mice 2, 28, and 56 days after Sh or Nx ( $n=4-6$ and 9-12 for Sh and Nx, respectively, in each strain at each time point). Data are shown as mean \pm SEM. ANOVA was followed by the Tukey-Kramer test. Nx versus Sh mice: ${ }^{*} P<0.05 ;{ }^{* * *} P<0.001$. FVB versus B6 mice: ${ }^{\# \#} P<0.001$. (D) Tubular cells proliferation index by nephron segment in FVB and B6 mice 2, 28, and 56 days after $\mathrm{Nx}$ ( $n=4 \mathrm{Nx}$ mice in each strain at each time point) using coimmunostaining of Ki-67 and specific tubular markers: lotus tetragonolobus lectin (LTL) for proximal tubules, Tamm-Horsfall (TH) for the ascending Henle loop and distal convoluted tubules, and dolichos biflorus agglutinin (DBA) for collecting tubules. Data are shown as mean \pm SEM. Mann-Whitney $U$ test. NxFVB versus NxB6: ${ }^{*} P<0.05$.

Consistent with the morphological data, 56 days after $\mathrm{Nx}$, renal function was preserved in B6 mice, whereas it was severely affected in FVB mice (Supplemental Figure 4). Similarly, urine protein and albumin excretion dramatically increased at day 56, exclusively in NxFVB mice (Supplemental Figure 4). As expected, mean arterial blood pressure was increased in NxFVB mice 56 days after nephron reduction compared with sham-operated controls and NxB6 mice $(138 \pm 11,110 \pm 2.5$, and $118 \pm 10 \mathrm{mmHg}$, respectively). However, the differences were not statistically significant.

To determine the relative contribution of the different nephron segments to the 2 waves of cell proliferation, we performed colocalization experiments using specific tubular markers and Ki-67. We observed that the first proliferative wave predominated in the proximal tubules and collecting ducts regardless of the genetic background (Figure 1D). In contrast, the second wave, observed only in NxFVB mice, involved mainly the proximal tubules and, to a minor extent, the Henle loops (Figure 1D).

Moreover, colocalization experiments using antibodies directed against proliferating cell nuclear antigen (PCNA), another marker of cell proliferation, and $\alpha$-smooth muscle actin ( $\alpha$-SMA), a marker of activated myofibroblasts, revealed that cell proliferation affected mainly tubular cells (Supplemental Figure 5). Notably, very few cells were stained by the 2 antibodies, indicating a low rate of fibroblast proliferation at day 56 .

Gene expression after $N x$ is driven in a strain- and time-dependent manner. To identify the genetic networks that trigger the compensatory and the deterioration processes, we next performed a temporal analysis of whole-kidney transcriptome in FVB and B6 mice at 2, 28, and 56 days after Nx or Sh (Supplemental Figure 6). Clustering by coinertia analysis of whole samples showed that gene expression was driven by 2 main components in a time-dependent manner (Figure 2A). The "strain effect" was the main determinant of renal gene expression, with FVB mice segregating in the upper part and B6 mice in the lower part of the panel. The second component was the "Nx effect," with Nx mice at day 2 being the greatest outlier from the Sh cluster in both strains. This partition was time dependent because NxB6 and NxFVB mice migrated toward the respective Sh cluster at day 28. Nevertheless, contrary to NxB6, NxFVB mice did not reconvert toward the respective ShFVB cluster at day 56 but rather moved in the opposite direction (Figure 2A). Remarkably, consistent with the changes of renal morphology and the pattern of cell proliferation, this unbiased analysis disclosed a 2-wave pattern of global gene expression in NxFVB and a single wave in NxB6 mice. The analysis of the amplitude of the differential gene expression further supported this pattern, with the observation that the most prominent differences between the 2 strains were at day 56 as compared with other time points (Figure 2B).

Gene and pathway enrichment analysis identifies a temporal modulation of gene expression after $N x$ with common and distinct patterns in FVB and B6 mice. To determine the temporal course of the genes and pathways modulated by $\mathrm{Nx}$, we compared $\mathrm{Nx}$ with Sh mice in each strain at each time point (Figure 3). A conspicuous number of genes were significantly upregulated or downregulated after $\mathrm{Nx}$ at each time point (Figure 3A). Nevertheless, the "Nx effect" was most remarkable at day 2 in both strains and at day 56 in the sensitive FVB strain. This was still the case when more stringent criteria (FDR $<0.01$; absolute $\log _{2}$ fold change $[F C] \geq 2$ ) were used to restrain the number of genes (Table 1). Interestingly, a dramatic decrease of the number of differentially expressed genes was observed at day 28, which was consistent with the return to a homeostatic state and the concept of a "quiescent phase." To determine the most relevant pathways $\mathrm{Nx}$ modulates, we then performed a gene set enrichment analysis (Figure $3 \mathrm{~B}$ ). A significant number of genes related to extracellular matrix and collagen synthesis and degradation were similarly induced by $\mathrm{Nx}$ in the 2 mouse strains from day 2 up to day 56 (Figure 3B). This was suggestive 
A

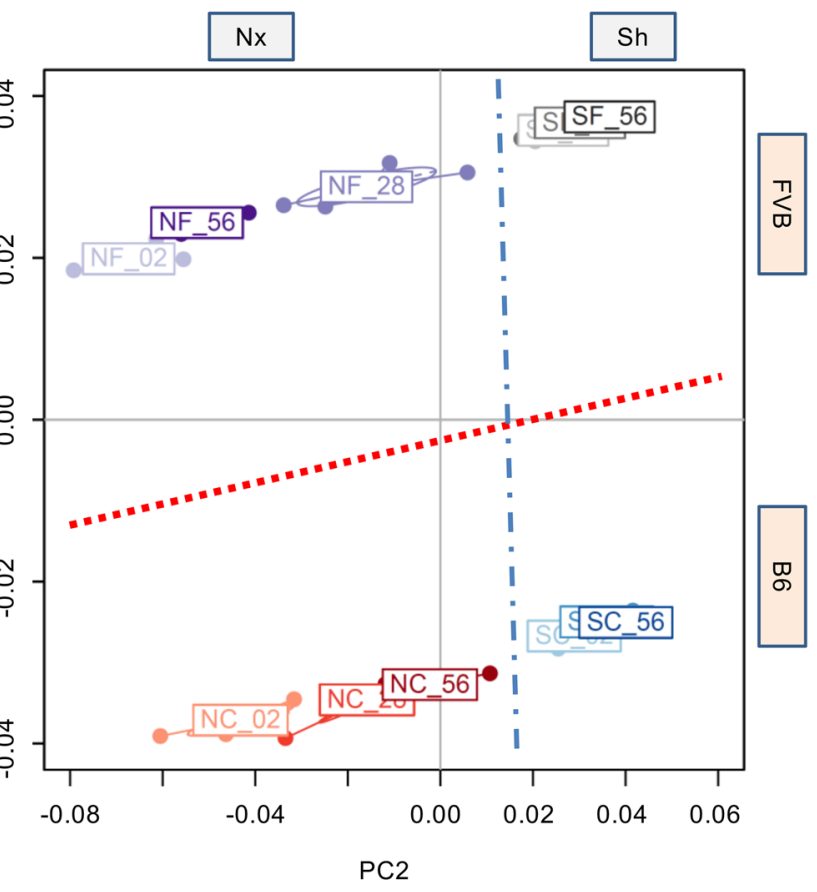

B

Day 2

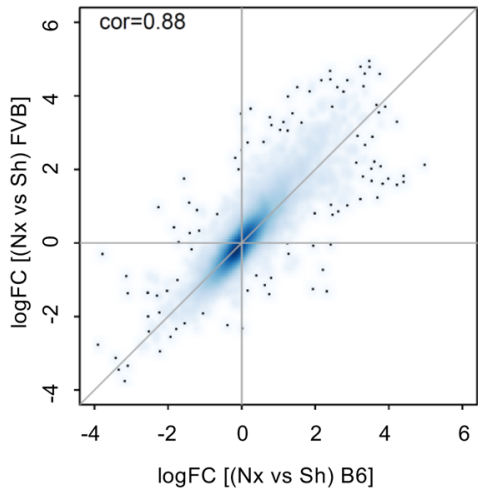

Day 28

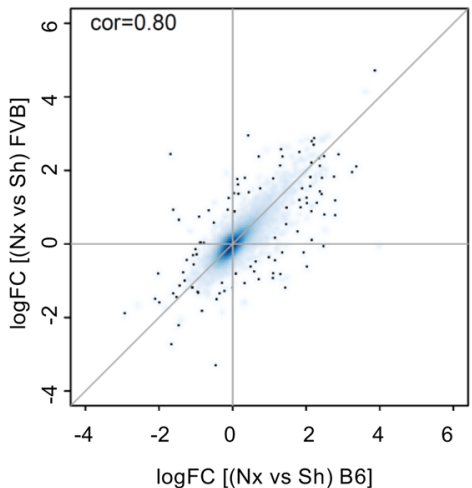

Day 56

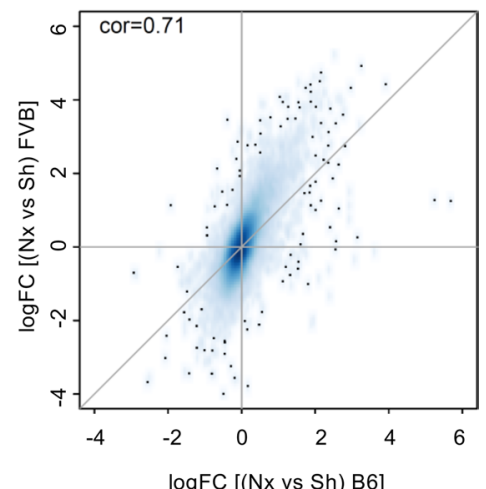

Figure 2. Temporal unbiased transcriptomic analysis revealed a distinct pattern of gene expression in sensitive and resistant strains. (A) Animal clustering using coinertia analysis. Sample partition by the 2 principal components (PCs): strain (dotted line, PC1) and surgery (dotted/dashed line, PC2); $n=4$ mice per group (S, sham operation; N, subtotal nephrectomy) from each strain (C, B6; F, FVB) at each time point (2, 28, and 56 days). (B) Scatter plots comparing the amplitude of differential gene expression between FVB ( $y$ axis) and B6 mice ( $x$ axis) 2, 28, and 56 days after sham operation (Sh) or subtotal nephrectomy (Nx). Each point represents a single gene. The correlation coefficient (cor) is indicated in each panel.

of a remodeling of the extracellular matrix in response to $\mathrm{Nx}$ but could also be the consequence of the surgical scar formation, particularly in the early phase. The deterioration phase was characterized by an upregulation of many gene sets specifically in the NxFVB mice, including members of EGFR, ERKs, RAS, and nonintegrin membrane extracellular matrix interaction pathways. Many cell cycle pathways were induced in both strains at day 2 but only in NxFVB mice at day 56. Nevertheless, and most remarkably, a number of genes related to mitosis and $\mathrm{G}_{1} / \mathrm{S}$ transition were more specifically upregulated in NxB6 mice than in NxFVB during the compensatory phase (Figure 3B).

Cell cycle progression differs between sensitive and resistant strains during compensatory growth. Because our finding pointed to potential differences in cell cycle progression between the 2 strains, we next investigated this phenomenon at days 2, 28, and 56 after Nx using colocalization experiments with Ki-67 and specific markers of S and $\mathrm{G}_{2} / \mathrm{M}$ cell cycle phases. Consistent with the transcriptomic analysis, we observed a higher proportion of cells in $\mathrm{S}$ and $\mathrm{G}_{2} / \mathrm{M}$ phases in NxB6 mice but exclusively 2 days after $\mathrm{Nx}$. By contrast, the number of cells in $\mathrm{G}_{1}$ phase was significantly higher in FVB mice than $\mathrm{B} 6$ mice 2 days after $\mathrm{Nx}$ (Figure $4 \mathrm{~A}$ ), suggesting a potential cell cycle $\mathrm{G}_{1} / \mathrm{S}$ blockade. Cell cycle progression was similar at days 28 and 56 after $\mathrm{Nx}$ in the 2 strains of mice. 
A
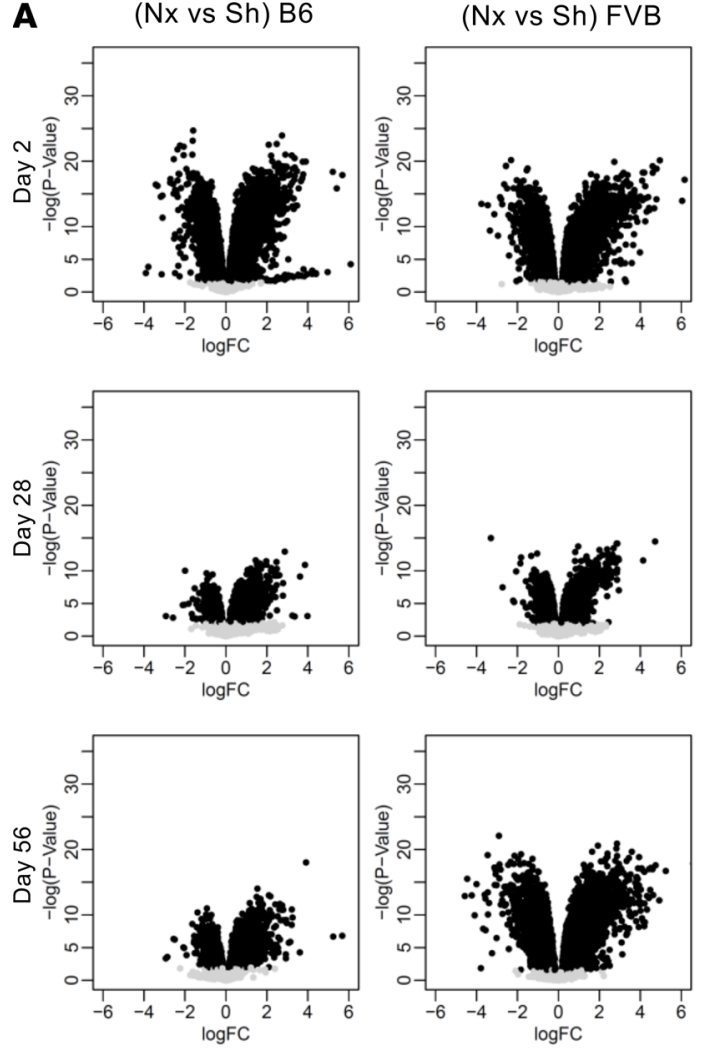

B

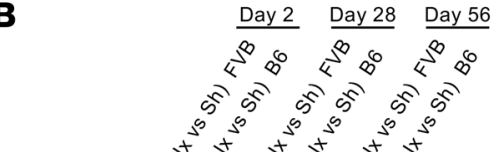

Enrichment score

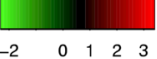

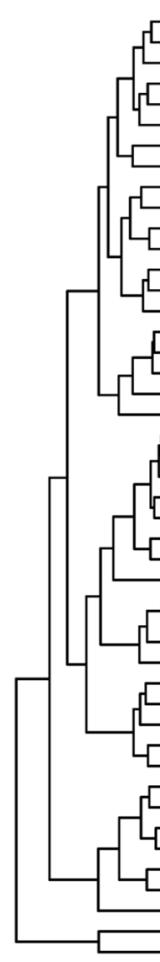

Extracellular matrix organization

Assembly of collagen fibrils and other multimeric structures Degradation of the extracellular matrix Condensation of Prometaphase Chromosomes Aurora B signaling

FOXM1 transcription factor network

Synthesis secretion and deacylation of Ghrelin Syndecan interactions

Activation of NIMA Kinases NEK9 NEK6 NEK7

Integrin cell surface interactions

A tetrasaccharide linker sequence is required for $G A G$ synthesis Chondroitin sulfate dermatan sulfate metabolism

Collagen degradation

Phosphorylation of Emi1

amb2 Integrin signaling

Collagen biosynthesis and modifying enzymes
Coling

Collagen formation

CRMPs in Sema3A signaling

Scavenging by Class A Receptors

Mitotic Metaphase and Anaphase

Mitotic Anaphase

Separation of Sister Chromatids

M Phase

Mitotic M M G1 phases

Mitotic Prometaphase

Resolution of Sister Chromatid Cohesion

Depolymerisation of the Nuclear amina

Nucleosome assembly

Deposition of new CENPA containing nucleosomes at the centromere

G1S Specific Transcription

Post chaperonin tubulin folding pathway

Signaling events mediated by PRL

EGFR Transactivation by Gastrin

Signalling to ERKs

Signalling to RAS

on integrin membrane ECM interaction

Peptide ligand binding receptors

G alpha i signalling events

Class A 1 Rhodopsin like receptors

GPCR ligand binding

Initial triggering of complement

Complement cascade

Integration of energy metabolism

Elevation of cytosolic Ca2+ levels

egulation of FZD by ubiquitination

Figure 3. Impact of nephron reduction on genes and pathways in FVB and B6 mice. (A) Volcano plots showing differentially expressed genes 2, 28, and 56 days after subtotal nephrectomy $(\mathrm{Nx})$ compared with sham operation (Sh) in B6 and FVB mice. (B) Heatmap of the top upregulated and downregulated pathways between Nx and Sh mice in FVB and B6 strains at days 2, 28, and 56. Expression values are represented in colors, where the range of colors shows the range of expression values (red for high and green for low).

Because p21 is a master regulator of the cell cycle, which has been implicated in the risk of developing renal lesions after $\mathrm{Nx}$, we next investigated whether changes in p21 expression might account for the differences observed between the 2 strains. Analysis of mRNA expression revealed that Cdkn1a was induced 2 days after $\mathrm{Nx}$ and that the increment was similar in FVB and B6 mice (Figure 4C). Nevertheless, immunohistochemistry showed that the protein was differentially expressed between the 2 strains. In fact, at day 2, p21 protein was significantly increased in FVB mice as compared with $\mathrm{B} 6$ mice in both $\mathrm{Nx}$ and $\mathrm{Sh}$ mice. More strikingly, the staining was prominently nuclear in FVB kidneys, particularly after Nx (Figure 4B), suggesting that a differential p21 nuclear translocation or stabilization may account for the cell cycle $\mathrm{G}_{1} / \mathrm{S}$ arrest in FVB mice during the compensatory growth.

We then wondered if these changes affected the renal compensatory growth process. Ki-67 staining revealed that many tubular cells were still proliferating at day 7 (Figure 4D). However, the rate of cell proliferation was significantly lower in NxFVB than in NxB6 mice (Figure 4D). Surprisingly, despite a lower proliferation rate, the kidney-to-body weight ratio was higher in NxFVB mice than in NxB6 mice (Figure 4E), suggesting that cell hypertrophy together with hyperplasia may account for kidney growth in NxFVB.

Gene expression analysis between sensitive and resistant strains identifies specific signaling signatures after $N x$. To identify the pathways that are selectively involved in the deterioration process, we next compared the Nx effect between FVB and $\mathrm{B} 6$ mice at each time point. To identify the most relevant candidates, we decided to use a stringent criterion, i.e., an FDR of less than 0.01 and absolute $\log _{2}$ FC of 2 or more. As expected, we observed that the expression of many genes 
Table 1. Nephrectomy effect on gene expression

\begin{tabular}{|c|c|c|c|c|}
\hline Time (Day) & Group & Up & Down & Total \\
\hline \multirow{2}{*}{2} & B6 & 182 & 33 & 215 \\
\hline & FVB & 276 & 25 & 301 \\
\hline \multirow{2}{*}{28} & B6 & 31 & 2 & 33 \\
\hline & FVB & 40 & 4 & 44 \\
\hline \multirow{2}{*}{56} & B6 & 49 & 4 & 53 \\
\hline & FVB & 266 & 63 & 329 \\
\hline
\end{tabular}

Number of significantly and differentially expressed genes 2, 28, and 56 days after Nx in B6 and FVB mice. The genes were considered significantly and differentially expressed when the FDR was less than 0.01 and the $|\log F C|$ was at least 2 .

changed after $\mathrm{Nx}$ in a similar way in both resistant and sensitive strains (compare Figure 3A and Table 1 with Figure 5A and Table 2). It is very likely that these genes reflect the common pathways involved in the compensatory processes Nx triggers. Remarkably, only a few genes were differentially expressed between NxFVB and NxB6 mice at each time point (Figure 5A and Table 2). A gene set enrichment analysis revealed a striking and early induction of type I IFN signature specifically in NxFVB mice at day 2 (Figure 5B). In contrast, no major and biologically relevant difference was observed at day 28 between NxFVB and NxB6 mice. However, many gene sets were selectively upregulated in NxFVB mice at day 56, including the EGFR, p38MAPK, and cell cycle pathways. Interestingly, several metabolic pathways, including gluconeogenesis, fatty acid $\beta$-oxidation, and citric acid TCA cycle, displayed a typical 2 -wave pattern in FVB mice. In fact, they were significantly downregulated in NxFVB mice at day 2 as compared with $\mathrm{NxB} 6$ and returned to baseline at day 28 , before being repressed again at day 56 (Figure 5B).

Type I IFN response is an early signature associated with later CKD progression. Because the induction of type I IFN response in FVB mice 2 days after $\mathrm{Nx}$ was particularly striking, we decided to better characterize this pathway. Using Ingenuity Pathway Analysis (QIAGEN) (16), we first observed that many upregulated genes in NxFVB at day 2 were type I IFN-stimulated genes that were interconnected with Irf7, a key transcriptional regulator of type I IFN-dependent immune response (Figure 6A). Quantitative reverse transcription PCR (RT-PCR) confirmed that many of these genes, including Ifi202b, Ifi204, Irf7, Usp18, and Isg15, were significantly upregulated 2 days after Nx, exclusively in FVB mice (Figure 6B). As previously reported, we observed that Ifi202b was undetectable in B6 mice (17). The temporal analysis of IFN-induced transcripts confirmed that this signature is an early and transient event occurring before the onset of renal lesions. In fact, the expression of most of these genes was shut down from 28 days after $\mathrm{Nx}$, and mRNA levels became similar to those of NxB6 (Supplemental Figure 7). Interestingly, we confirmed the early activation of type I IFN response in another widely used experimental model of CKD, the unilateral ureteral obstruction (UUO) (Supplemental Figure 8). In fact, a time course analysis revealed that IFN targets increased from 7 days after UUO before the development of overt glomerular lesions. However, at variance with $\mathrm{Nx}$, the response endured over time in this model (Supplemental Figure 8).

Type I IFN response correlates with an imbalance between silent and immunogenic cell deaths and immune cell activation. We next investigated which cellular events account for the induction of type I IFN response in NxFVB. It is known that DNA damage accompanies acute tubular necrosis, a lesion that we observed 2 days after Nx. Because DNA damage may trigger type I IFN expression, we next studied the expression of phospho-histone H2AX (p-H2AX), a marker of DNA damage. Immunohistochemistry and Western blot experiments revealed a substantial increase of $\mathrm{p}-\mathrm{H} 2 \mathrm{AX}$ expression 2 days after $\mathrm{Nx}$. However, the increase was comparable in NxFVB and NxB6 mice (Figure 7A). Because cell death and immunogenic cell death may also trigger type I IFN expression, we next investigated the balance between silent and immunogenic cell death. Interestingly, we observed that expression of Ripk3 and its downstream target Mlkl, 2 markers of necroptosis, was significantly increased in FVB mice 2 days after Nx (Figure 7B). In contrast, we did not detect any major difference in apoptosis between the 2 strains, as judged by TUNEL staining (Figure 7C). Nevertheless, we observed a strong induction of kidney injury molecule-1 (KIM1), a molecule involved in the clearance of apoptotic cells, but predominantly in $\mathrm{B} 6$ mice (Figure 7, D and E). Together, these data suggest that reduced clearance of apoptotic debris in NxFVB mice might result in an immunogenic response in FVB mice. Consistent with this idea, we showed that plasmacytoid dendritic cells (pDCs), 
A
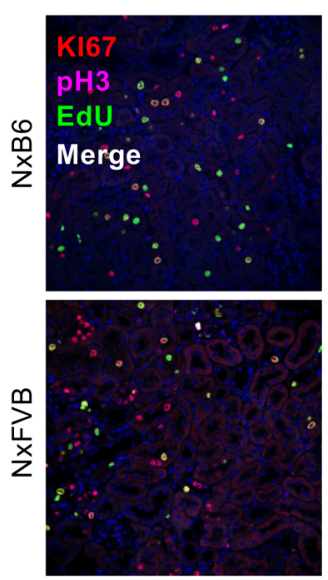

Day 28
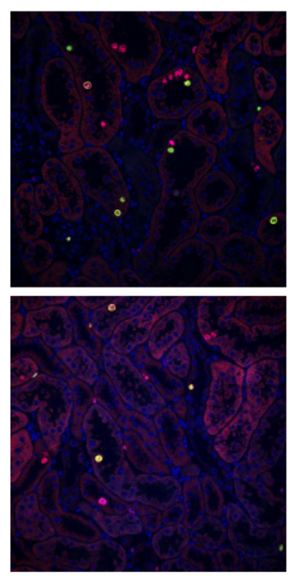

Day 56

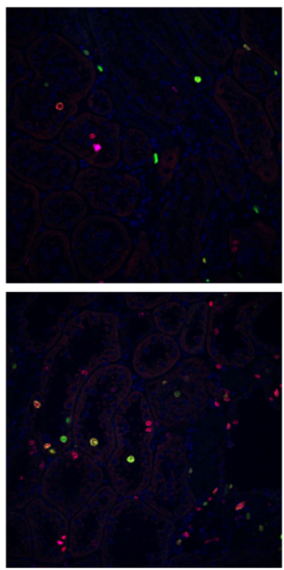

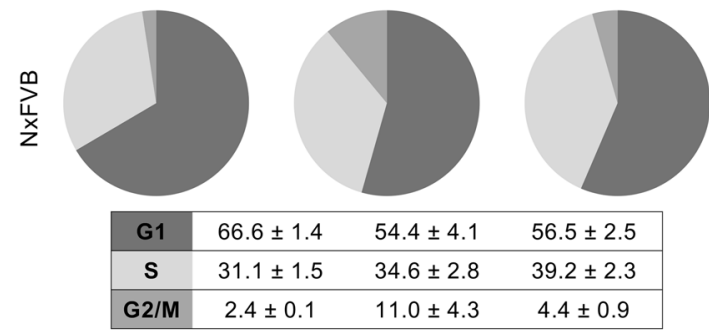

B

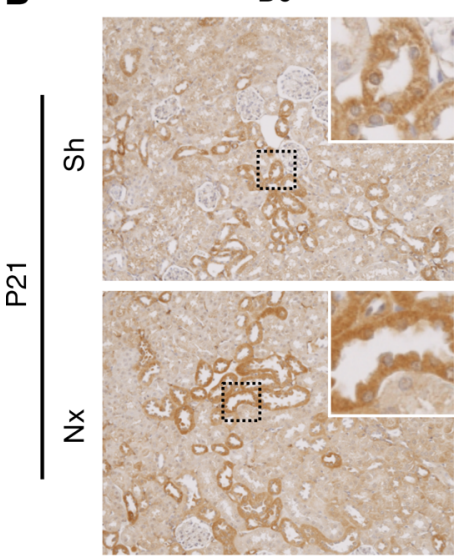

FVB

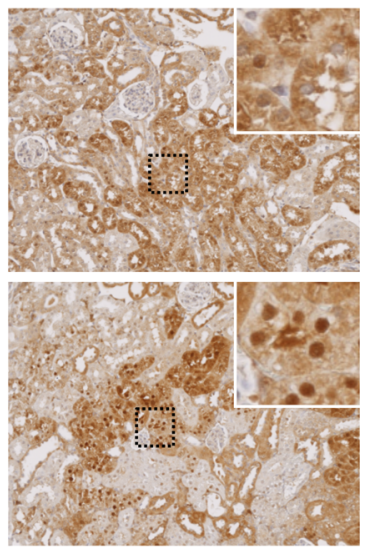

C

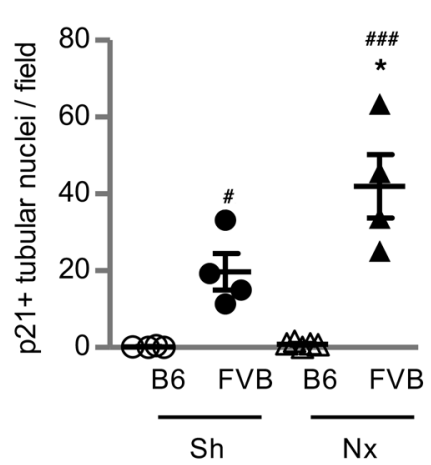

Cdkn1a
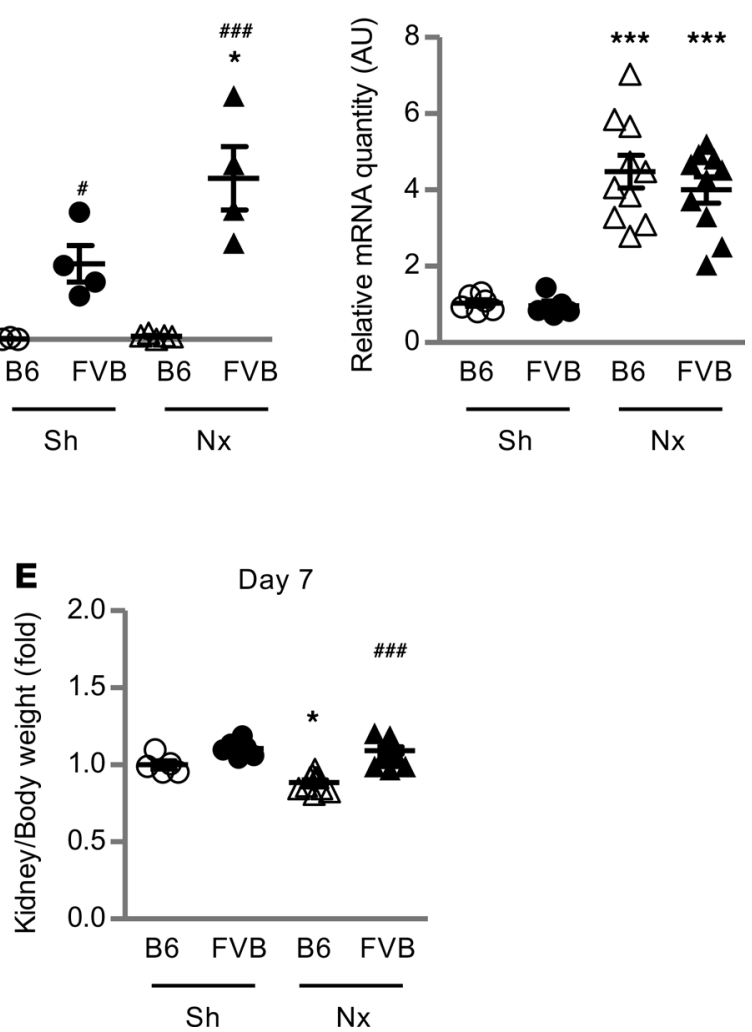

Day 7
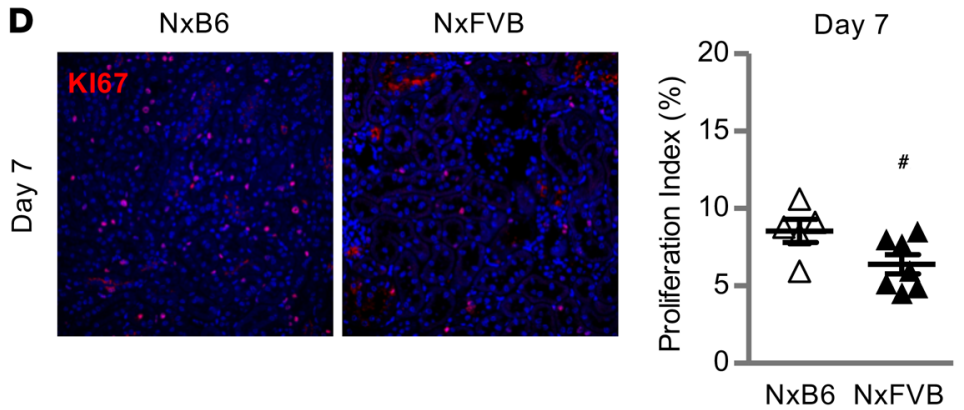

Day 2

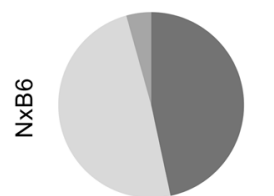

\begin{tabular}{|c|ccc|}
\hline G1 & $46.7 \pm 6.9$ & $47.7 \pm 8.4$ & $51.4 \pm 3.8$ \\
\hline S & $49.0 \pm 7.2$ & $43.5 \pm 5.5$ & $39.4 \pm 5.5$ \\
\hline G2/M & $4.3 \pm 0.5$ & $8.8 \pm 3.5$ & $9.2 \pm 2.3$ \\
\hline
\end{tabular}

Day 28

Day 56

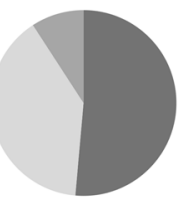

$$
\text { (1) }
$$


A
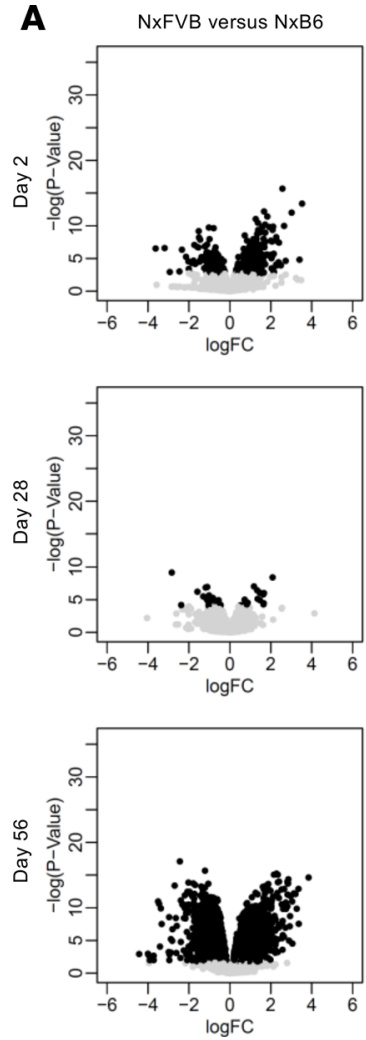

B
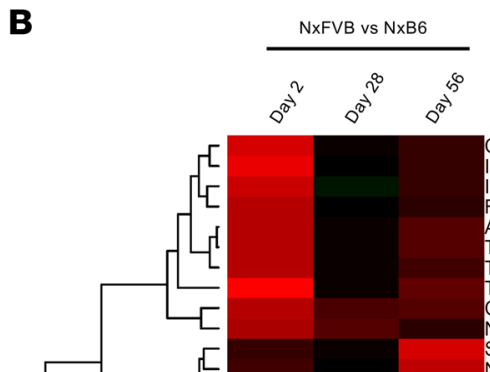

$\begin{array}{llll}-3 & -1 & 1 & 3\end{array}$

CRMPs in Sema3A signaling

nterferon alpha beta signaling

L27 mediated signaling events

Regulation of IFNA signaling

Activation of IRF 3 IRF7 mediated by TBK1 IKK epsilon

RRAF6 mediated IRF7 activation

TRAF3 dependent IRF activation pathway

TRAF6 mediated IRF7 activation in TLR7 8 or 9 signaling

CHL1 interactions

Nectin $\mathrm{Necl}$ trans heterodimerization

Signalling to RAS

Non integrin membrane ECM interactions

Signaling events mediated by PRL

Syndecan interactions

p38MAPK events

EGFR Transactivation by Gastrin

Alpha6Beta4Integrin

Syndecan 3 mediated signaling events

Elevation of cytosolic $\mathrm{Ca} 2+$ levels

Retinoid cycle disease events

The canonical retinoid cycle in rods twilight vision

Cytosolic iron sulfur cluster assembly

Neurotransmitter Clearance In The Synaptic Cleft

regulation of FZD by ubiquitination

iile salt and organic anion SLC transporters

Class $\mathrm{C} 3$ Metabotropic glutamate pheromone receptors

Lysine catabolism

Branched chain amino acid catabolism

Propionyl COA catabolism

Creatine metabolism

Formation of ATP by chemiosmotic coupling

Mitochondrial Fatty Acid Beta Oxidation

Pyruvate metabolism and Citric Acid TCA cycle

Citric acid cycle TCA cycle

The citric acid TCA cycle and respiratory electron transport

Beta oxidation of octanoyl COA to hexanoyl CoA

Beta oxidation of decanoyl CoA to octanoyl CoA CoA

Beta oxidation of pristanoyl COA

Respiratory electron transport

Respiratory electron transport ATP synthesis by chemiosmotic coupling

Figure 5. Genes and pathways are differentially modulated between the $\mathbf{2}$ strains in response to nephron reduction. (A) Volcano plots showing differentially expressed genes between FVB and B6 2, 28, and 56 days after subtotal nephrectomy (Nx) using sham-operated (Sh) animals in each strain and at each time point as controls. (B) Heatmap of the top upregulated and downregulated pathways between NxFVB and NxB6 mice using Sh animals as controls at day 2, 28, and 56. Expression values are represented in colors, where the range of colors shows the range of expression values (red for high and green for low).

known to produce large amounts of type I IFN in almost all the tissues, were strongly upregulated in FVB mice 2 days after $\mathrm{Nx}$, as judged by the massive increase of SiglecH and Tlr7, 2 markers of pDCs (Figure $8 \mathrm{~A}$ ). We also observed a significant increase of CD3, a marker of T lymphocytes, exclusively in FVB mice 2 days after $\mathrm{Nx}$ (Figure $8, \mathrm{~B}$ and $\mathrm{C}$ ). In contrast, macrophage infiltration significantly increased in both B6 and FVB mice 2 days after $\mathrm{Nx}$ (Figure 8, D and $\mathrm{E}$ ). Together, these results point to a distinct profile of renal inflammatory cells in kidneys of FVB mice, which could account for the specific type I IFN pathway activation in the early phase after Nx. However, this pattern seems to be specific to the compensatory phase. In fact, a careful time course quantification of infiltrating inflammatory cells revealed that $\mathrm{CD}^{+}$cells increased the same magnitude in B6 and FVB mice from day 28 after $\mathrm{Nx}$, whereas $\mathrm{F} 4 / 80^{+}$cells exhibited a 2-wave pattern in both B6 and FVB mice (Supplemental Figure 9). It is worth noting that Ly6B, a marker of neutrophils, was almost undetectable in both $\mathrm{Sh}$ and $\mathrm{Nx}$ mice, regardless of the genetic background and the experimental time point (data not shown).

Early activation of type I IFN response results in albuminuria and glomerular lesions in initially resistant mice. Finally, we tested the hypothesis that the molecular pathways activated during the compensatory phase, i.e., the type I IFN response, may be critically involved in later lesion development. To test this hypothesis, we injected poly(I:C), a potent activator of TLR3 and inducer of type I IFN signature in initially resistant NxB6 mice. Poly(I:C) was injected every 48 hours during the first week after the first Nx (Figure 9A). We used this protocol because we previously showed that this schema of injection resulted in a transient induction of IFN targets that returned to baseline levels after 1 week (our unpublished observations). Consistently, we confirmed that, in this study, the expression of Irf7 and SiglecH was upregulated 7 days after the first Nx only in the kidney of mice treated with poly(I:C) (Figure 9B). As expected, vehicle-treated B6 mice did not develop renal lesions after Nx. Remarkably, however, poly(I:C)-treated mice developed signifcant glomerular lesions 16 weeks after $\mathrm{Nx}$ (Figure 9C). Lesions consisted mainly of mesangial expansion, which was particularly severe at the vascular pole. Notably, $62 \%$ of glomeruli displayed moderate to severe 
Table 2. Nephrectomy and strain effect on gene expression

\begin{tabular}{cccccc}
\hline Time (Day) & Group & Up & Down & Total \\
2 & FVB vs. B6 & 19 & 4 & 23 \\
28 & FVB vs. B6 & 1 & 1 & 2 \\
56 & FVB vs. B6 & 49 & 34 & 83
\end{tabular}

Number of significantly and differentially expressed genes between NxFVB and NxB6 using Sh as a control at 2, 28, and 56 days after Nx. The genes were considered significantly and differentially expressed when the FDR was less than 0.01 and the $|\log F C|$ was at least 2.

lesions (score 2 or 3 ) in poly(I:C)-treated $\mathrm{Nx}$ mice, whereas $58 \%$ of glomeruli displayed no or very mild lesions (score 0 or 1 ) in placebo-treated $\mathrm{Nx}$ animals. Consistently, albuminuria was significantly increased from 8 weeks after Nx (Figure 9, D and E). Surprisingly, the tubulointerstitial compartment appeared grossly normal. Together, these data show that the genetic programs activated during the compensatory growth may predispose mice to later glomerular lesion development.

\section{Discussion}

In the present study, using unbiased profiling of whole-kidney transcriptome in mouse strains that differentially react to nephron reduction, we showed that gene expression after $\mathrm{Nx}$ was driven in a strainand time-dependent manner. Our results supported previous published findings on the deleterious role of EGFR pathway activation and metabolic pathway repression in CKD progression $(7,8,13,18,19)$. Most remarkably, we showed that the early events occurring during the compensatory renal growth phase may dramatically influence the risk of developing progressive CKD. In particular, we observed that alterations of cell cycle progression, notably a $\mathrm{G}_{1} / \mathrm{S}$ cell cycle arrest and $\mathrm{p} 21$ nuclear translocation during the renal compensatory phase, correlated with later renal deterioration. We also identified type I IFN response as an early and potentially previously unrecognized signature associated with later CKD development. More importantly, we demonstrated that the induction of a transient type I IFN response during the compensatory period in initially resistant B6 mice predisposes to lesion development. Collectively, our results reveal that the early cellular and molecular events occurring right after a renal injury may account for the risk of developing renal lesions later.

Because de novo nephrogenesis does not occur in mammals, compensatory growth of the remnant kidney can result only from a combination of tubular cell hyperplasia (i.e., increase in cell number) and hypertrophy (i.e., increase in cell volume) $(3,5)$. Because both processes require that the cell enters into the cell cycle, the balance between cell hypertrophy and hyperplasia must be tightly regulated. Interestingly, our transcriptional data suggested a potential imbalance in cell cycle progression during the early period after $\mathrm{Nx}$, with a potential $\mathrm{G}_{1} / \mathrm{S}$ cell cycle arrest in FVB mice. This change was associated with the nuclear translocation of $\mathrm{p} 21$, a well-known regulator of the $\mathrm{G}_{1} / \mathrm{S}$ checkpoint. In a noteworthy addition to this observation, Megyesi et al. showed that p21-knockout mice were protected from CKD after Nx and suggested that an imbalance between renal hypertrophy and renal hyperplasia may predispose to CKD (20). In agreement with this idea, we observed that the increase of kidney weight in the lesion-prone FVB mice was very likely associated with cell hypertrophy because cell proliferation decreased between 2 and 7 days after $\mathrm{Nx}$ as compared with the resistant B6 mice. One intriguing observation of our study is the absence of a cell cycle $\mathrm{G}_{2} / \mathrm{M}$ arrest during lesion development. In fact, previous studies reported that cell cycle $\mathrm{G}_{2} / \mathrm{M}$ arrest is associated with the development of interstitial fibrosis in various models of acute kidney injury $(15,21)$. Whether differences in the experimental models may account for this discrepancy deserves further study.

In the present study, we identified type I IFN response as an early signature associated with CKD onset. Type I IFNs, including IFN- $\alpha$ and IFN- $\beta$, are central regulators of antiviral immunity, but little is known about their roles in kidney diseases (22-24). IFN- $\alpha$ overexpression correlates with renal lesions, whereas IFN receptor deficiency abrogates autologous nephrotoxic serum nephritis (25). Inhibition of IFN receptors also attenuates glomerular lesions in lupus nephritis (26). On the other hand, it has been reported that type I IFN infusion induces podocyte and glomerular lesions in a model of adriamycin nephropathy (27) as well as proteinuria in APOL1-transgenic mice (28). In the present study, we demonstrated that type I IFN induction in initially resistant mice was associated with delayed but significant glomerular lesions and albuminuria (Figure 9, C and E). In line with this observation, IFN administration to newborn mice 
A

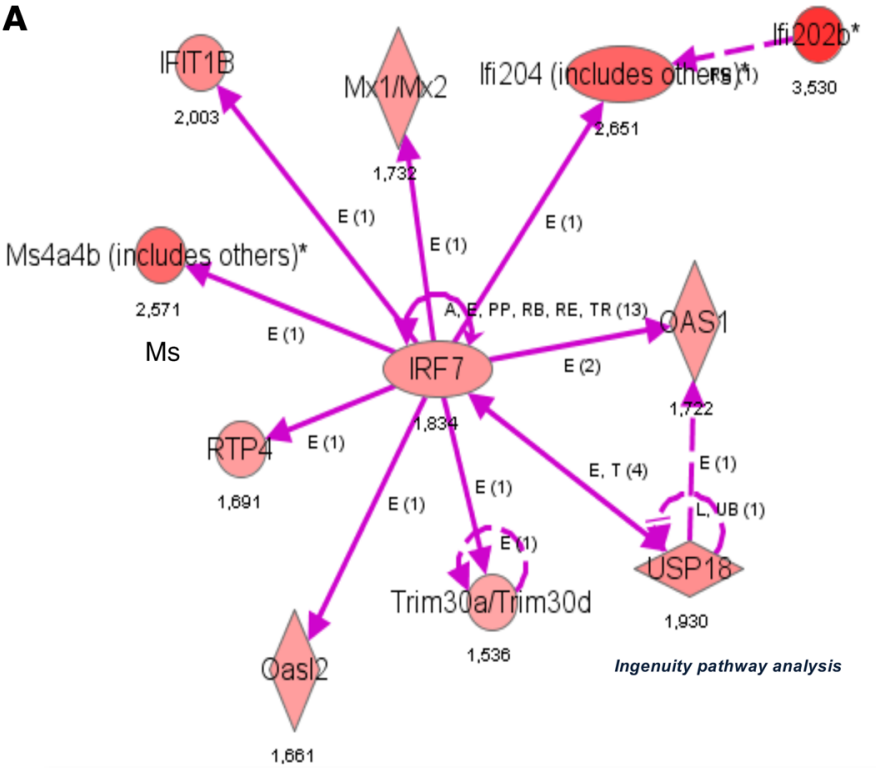

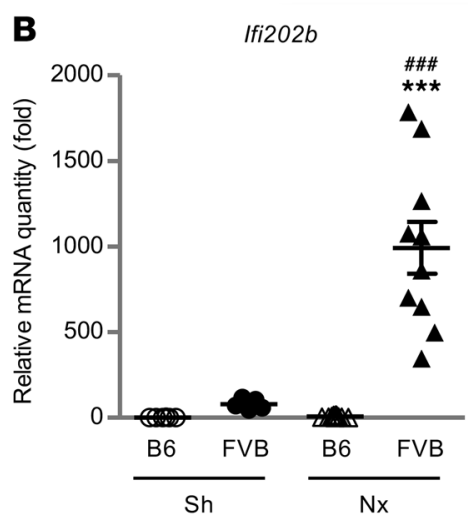

$\operatorname{lsg} 15$

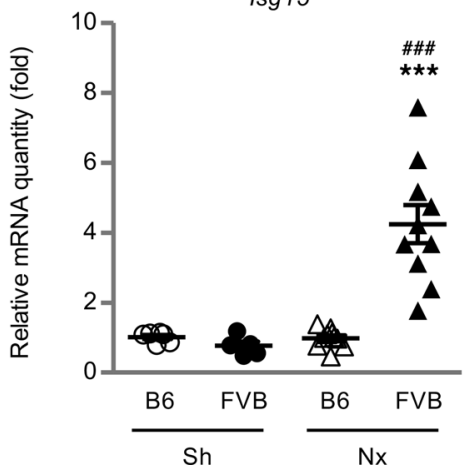

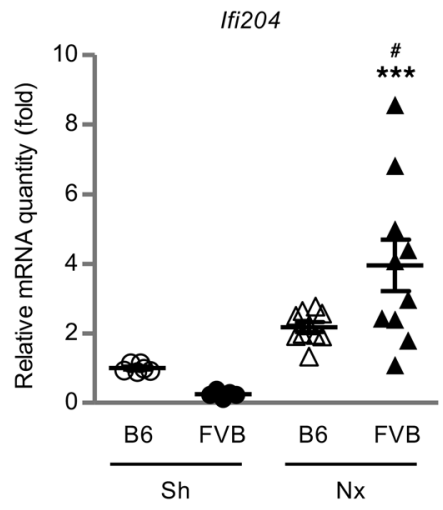

Usp18

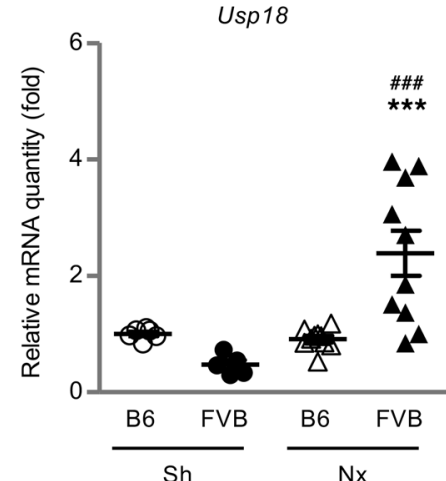

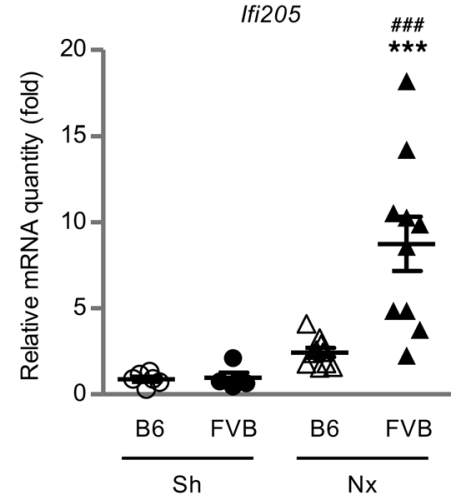

Irf7

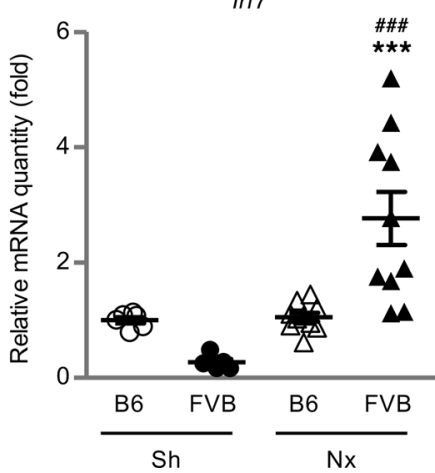

Figure 6. Nephron reduction results in type I IFN response in FVB mice during compensatory growth. (A) Ingenuity Pathway Analysis showing a cluster of upregulated and interconnected type I IFN-induced genes in FVB compared with B6 mice 2 days after subtotal nephrectomy ( $\mathrm{Nx}$ ) using sham-operated (Sh) animals as controls. (B) mRNA expression of Ifi202b, Ifi204, Ifi205, Isg15, Usp18, and Irf7 in FVB and B6 mice 2 days after Sh or Nx ( $n=5-6$ and 10 for Sh and Nx, respectively, in each strain). Data are shown as mean \pm SEM. ANOVA was followed by the Tukey-Kramer test. Nx versus Sh mice: ${ }^{* * *} P<0.001$. FVB versus B6 mice: ${ }^{*} P<0.05 ; \# \# \#<0.001$

during the first week of life results in glomerulonephritis 8 months later (29). The delay required between type I IFN exposure and the onset of renal lesions is very intriguing. It is worth noting that in both conditions IFNs act in a context of high tubular cell proliferation. Whether IFNs may prime proliferating cells to activate later genetic programs that finally trigger lesion development is an exciting hypothesis that requires further investigation. 
A

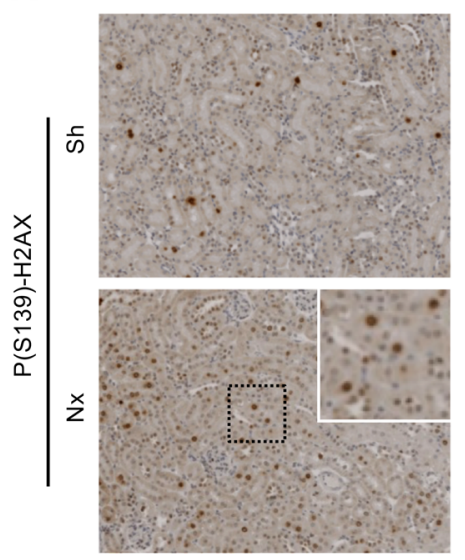

B

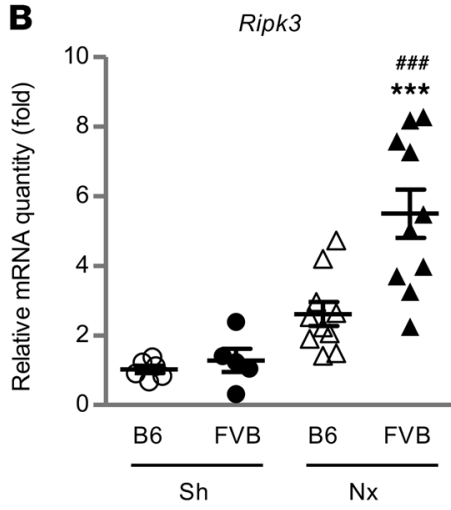

FVB
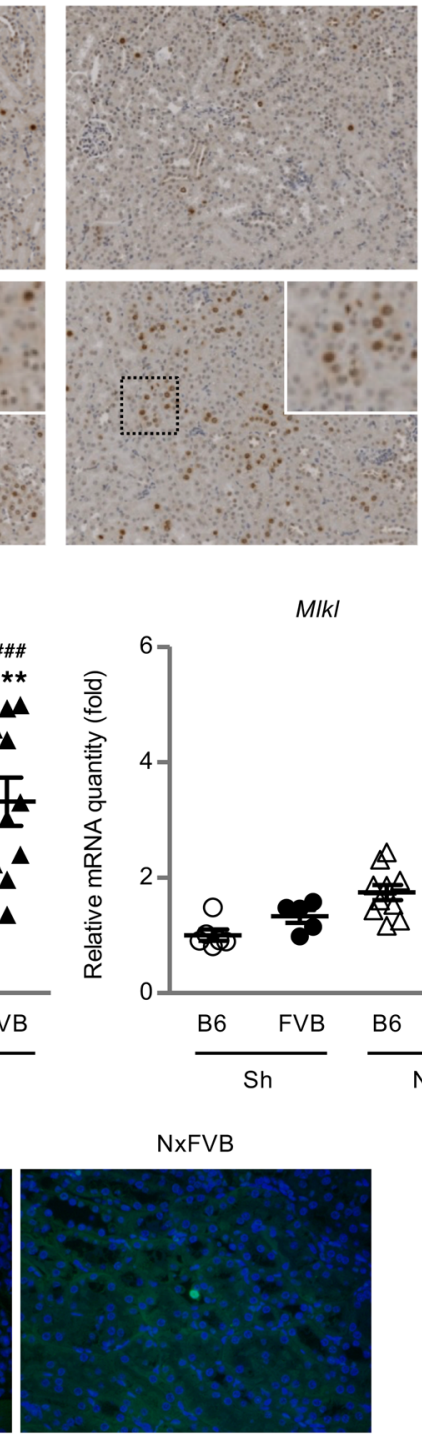

FVB

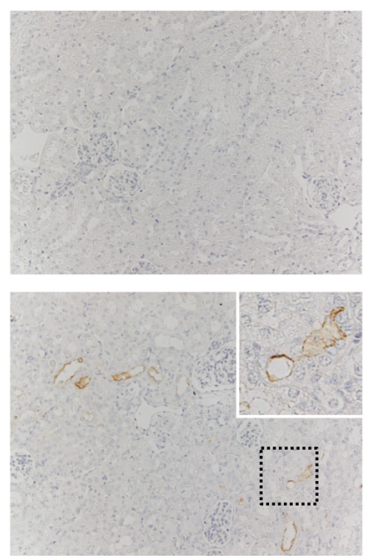

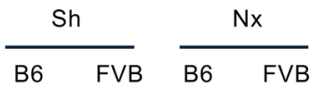

$\mathrm{P}(\mathrm{S} 139) \mathrm{H} 2 \mathrm{AX}$

GAPDH

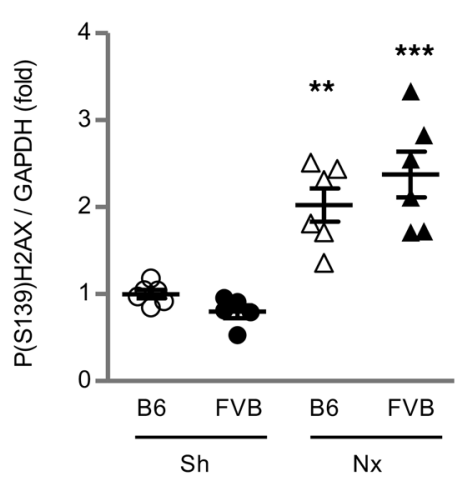

C

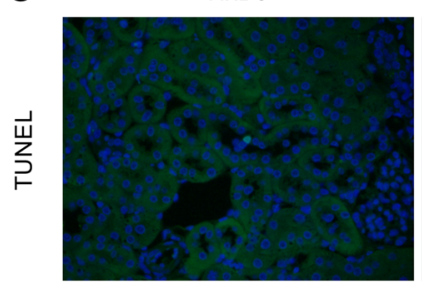

B6

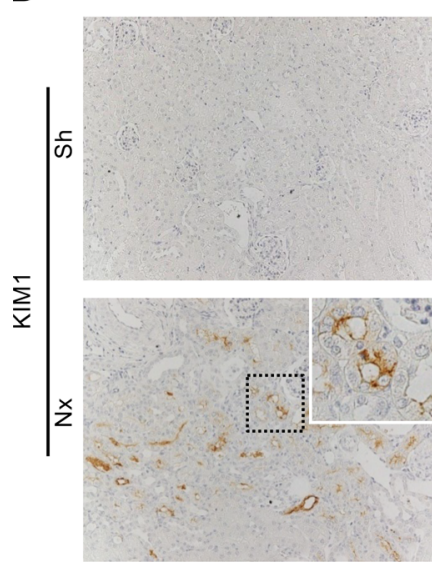

Figure 7. Different patterns of tubular cell death in sensitive and resistant strains after nephron reduction. (A) Analysis of phospho-histone $\mathrm{H} 2 \mathrm{~A} . \mathrm{X}$ (Ser139) expression by immunohistochemistry (left, original magnification, $\times 200$, inset $2 \times ; n=4$ mice per group in each strain) and Western blot (right) in FVB and B6 mice 2 days after sham operation (Sh) or subtotal nephrectomy ( $\mathrm{Nx}$ ) ( $n=5-6$ mice per group in each strain). Data are shown as mean \pm SEM. ANOVA was followed by the Tukey-Kramer test. Nx versus Sh mice: ${ }^{* *} P<0.01 ;{ }^{* *} P<0.001$. (B) Analysis of Ripk3 and Mlkl mRNA expression in FVB and B6 mice 2 days after Sh or Nx ( $n=5-6$ and 10 for Sh and Nx mice, respectively, in each strain). Data are shown as mean \pm SEM. ANOVA was followed by the Tukey-Kramer test. Nx versus Sh mice: ${ }^{* *} P<0.001$. FVB versus B6 mice: ${ }^{* \#} P<0.001$. (C) Representative images (left) 
and quantification (right) of TUNEL apoptosis assay in FVB and B6 mice 2 days after Nx ( $n=8$ mice per group). Data are shown as mean \pm SEM. (D) Representative images (left) and quantification (right) of KIM1 expression in B6 and FVB mice 2 days after Sh or Nx (original magnification, $\times 200$, inset $3 x ; n=4$ mice, 5 for Sh and Nx, respectively, in each strain). Data are shown as mean \pm SEM. ANOVA was followed by the Tukey-Kramer test. Nx versus Sh mice: ${ }^{*} P<0.05$; ${ }^{* *} P<0.001$. FVB versus B6 mice: ${ }^{*} P<0.05$. (E) Kim1 mRNA expression in FVB and B6 mice 2 days after Sh or Nx ( $n=$ 5-6 and 10 for Sh and Nx, respectively, in each strain). Data are shown as mean \pm SEM. ANOVA was followed by the Tukey-Kramer test. Nx versus Sh mice: ${ }^{* *} P<0.001$. FVB versus B6 mice: ${ }^{\# \#} P<0.001$.

The original source of type I IFNs in the setting of $\mathrm{Nx}$ is difficult to ascertain because almost any cell type may produce type I IFNs upon stimulation (23, 30-32). Tubular cells may contribute to IFN production in a cell-autonomous manner $(33,34)$. Alternatively, lymphocytes, which we found selectively increased in FVB mice 2 days after Nx, may participate in IFN production. IFN response may also result from $\mathrm{pDC}$ activation. Indeed, tubular cell damage can trigger $\mathrm{pDC}$ activation through the release of cellular debris in the extracellular milieu, whereby the danger signals are recognized to initiate immune responses (24). In line with these observations, the potential imbalance between immunogenic and silent tubular cell death that we observed in remnant kidneys of FVB mice may contribute to activate pDCs. Although apoptosis per se is not considered an immunogenic cell death, a defect in apoptotic cell clearance may also affect inflammatory responses (24). Interestingly, we observed that although apoptosis was similar in B6 and FVB remnant kidneys, the expression of KIM1, a molecule known to clear apoptotic cells, was dramatically increased but exclusively in $\mathrm{B} 6$ mice (35). Hence, it is conceivable to propose that because of the absence of KIM1 activation, the accumulation of apoptotic debris in FVB mice leads to cell apoptosisrelated immunogenicity, innate immunity, renal inflammation, and finally IFN response.

We previously discovered that a hypomorphic variant of Mitfa, a gene encoding for a helix-loop-helix transcription factor, accounts at least in part for the increased susceptibility of FVB mice to renal lesions (14). In contrast, to the best of our knowledge, no polymorphism within the IFN genes has been reported to predispose to CKD. Only a microdeletion of Ifi202b gene was described in B6 mice (17). Whether IFN is a target of MITF-A is an interesting hypothesis that deserves further study.

Type I IFNs drive many cellular responses to stress and may notably activate the p53/p21 axis to inhibit cell proliferation and induce cell death or senescence $(34,36)$. IFN- $\beta$ enhances p21 nuclear translocation and contributes to preventing tumorigenesis $(37,38)$. In the kidney, IFN- $\alpha$ has been shown to induce parietal epithelial cell cycle arrest in the $G_{1}$ phase through the induction of $p 21$, which in turn impairs podocyte repair (27). Consistently, in the present study, we observed $G_{1}$ arrest and p21 nuclear translocation in NxFVB mice during the first wave of cell proliferation, when IFN response was activated. Thus, it is possible that type I IFN response may exert an inhibitory effect on cell growth in the setting of Nx, notably through the induction of $\mathrm{p} 21$ and cell cycle arrest $(36,39)$.

Surprisingly, poly(I:C) administration in NxB6 mice led to glomerular lesions but did not affect the tubulointerstitial compartment that appeared grossly normal. On the contrary, FVB mice displayed both glomerular and tubulointerstitial lesions after Nx. This discrepancy could be explained by the fact that with the protocol used (Figure 9A), poly(I:C) induced only a mild activation of IFN response. In fact, it is known that the initial stimuli and cellular source may greatly influence the nature, intensity, duration, and potential effects of type I IFN production. Alternatively, it is also possible that different signaling pathways affect different nephron compartments. We have previously shown that EGFR activation is critically involved in the development of tubulointerstitial lesions in FVB mice after $\mathrm{Nx}(7,8,14)$. Remarkably, we observed that poly(I:C) infusion did not lead to any activation of EGFR in NxB6 mice (data not shown), which might be in favor of this hypothesis.

In conclusion, the present study revealed that the induction of type I IFN response and perturbations of cell cycle progression during the early phase after nephron reduction critically determine the risk of maladaptive renal compensatory growth and progression toward CKD. Hence, the activation of specific molecular signatures during the early phase after nephron reduction might define the fate of remaining nephrons toward compensation or deterioration.

\section{Methods}

Animals. All experiments were performed on 9-week-old female mice from C57BL6/J (B6) and FVB/N (FVB) strains (Janvier's Lab). Animals were fed ad libitum and housed in a room with constant ambient temperature and a 12-hour light/12-hour dark cycle. 

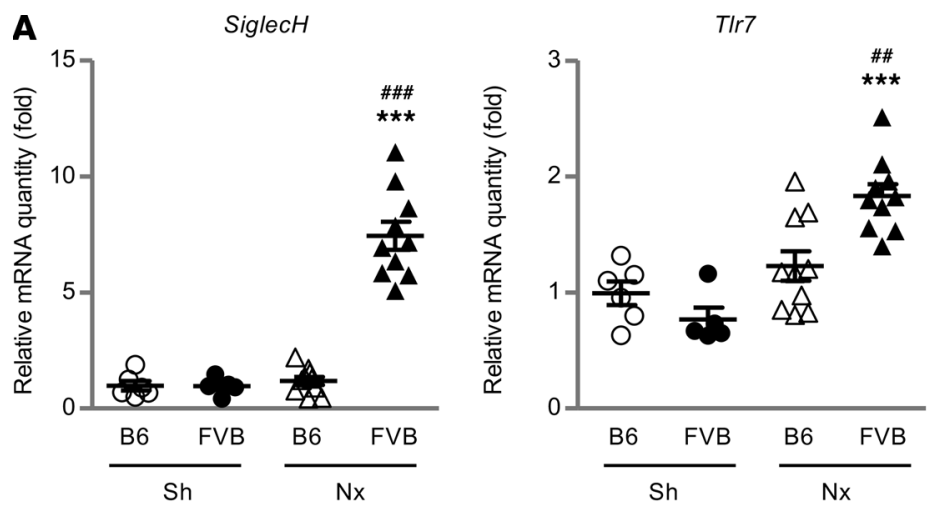

B

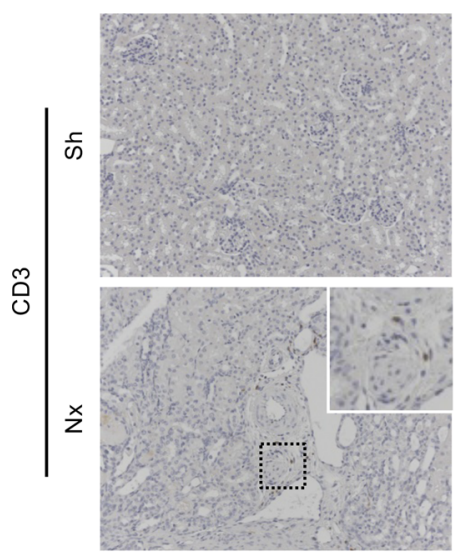

D

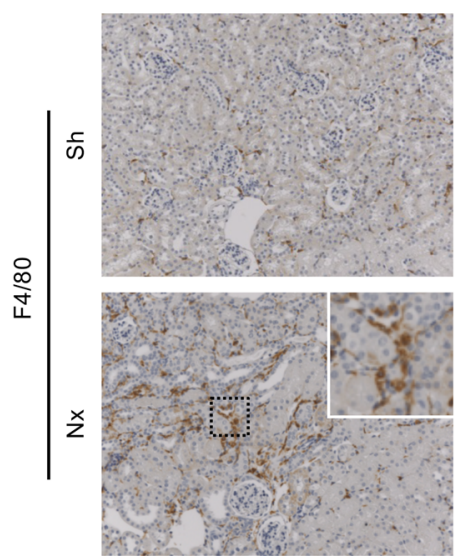

FVB

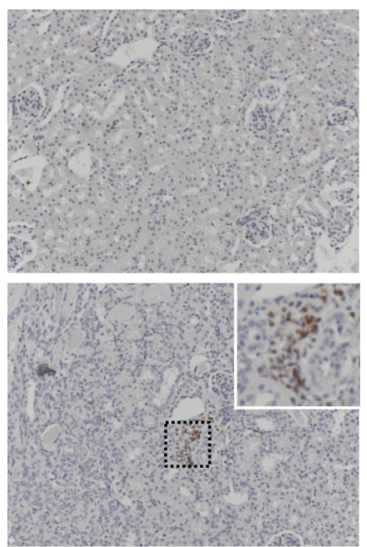

FVB

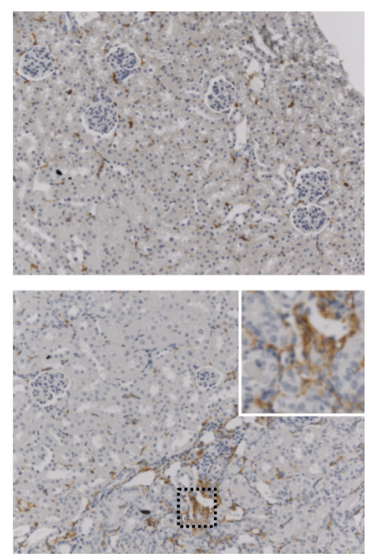

Day 2

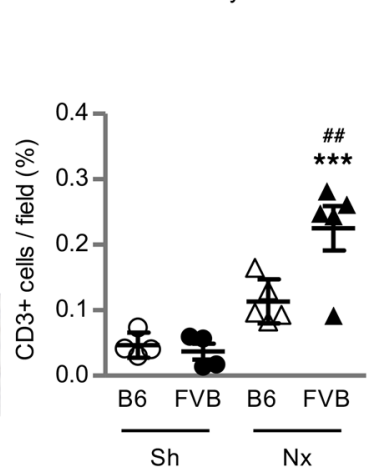

Day 2

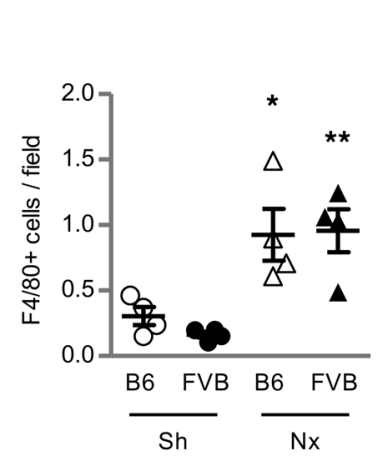

C

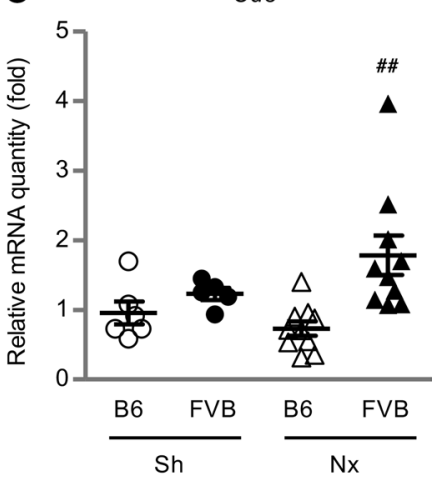

E

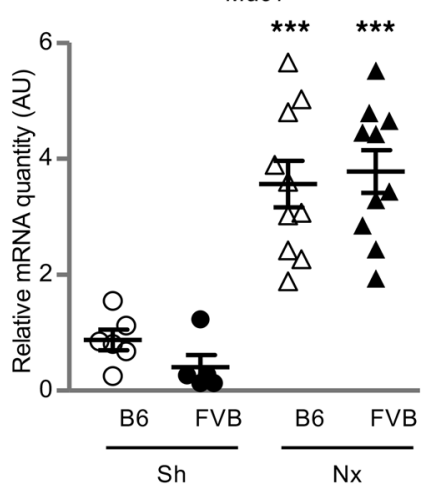

Figure 8. Inflammatory cell markers are increased in the sensitive FVB mice $\mathbf{2}$ days after nephron reduction. (A) Analysis of plasmacytoid dendritic cell markers using SiglecH and T/r7 mRNA expression in B6 and FVB mice 2 days after sham operation (Sh) or subtotal nephrectomy (Nx) ( $n=5-6$ and 10 for Sh and Nx, respectively, in each strain). Data are shown as mean \pm SEM. ANOVA was followed by the Tukey-Kramer test. Nx versus Sh mice: ${ }^{* * *} P<0.001$. FVB versus B6 mice: ${ }^{\#} P<0.01$; \#\#\# $<0.001$. (B) Representative images (left) and quantification (right) of T lymphocyte infiltration in B6 and FVB mice 2 days after Sh or Nx by CD3 immunohistochemistry (original magnification, $x 200$, inset $2 \times ; n=4$ and 5 for Sh and Nx, respectively, in each strain) Data are shown as means \pm SEM. ANOVA was followed by the Tukey-Kramer test. Nx versus Sh mice: ${ }^{* * *} P<0.001$. FVB versus B6 mice: ${ }^{\#} P<0.01$. (C) Cd3 mRNA expression ( $n=5-6$ and 10 for Sh and Nx, respectively, in each strain). Data are shown as mean \pm SEM. ANOVA was followed by the Tukey-Kramer test. FVB versus B6 mice: \#\# $P<0.01$. (D) Representative images (left) and quantification (right) of macrophage infiltration in B6 and FVB mice 2 days after Sh or Nx by F4/80 immunohistochemistry (original magnification, $\times 200$, inset $2 \times ; n=4$ and 5 for Sh and $\mathrm{Nx}$, respectively, in each strain) Data are shown as mean \pm SEM. ANOVA was followed by the Tukey-Kramer test. Nx versus Sh mice: ${ }^{*} P<0.05$; ${ }^{* *} P<$ 0.01. (E) Mac1 mRNA expression ( $n=5-6$ and 10 for Sh and Nx, respectively, in each strain). Data are shown as mean \pm SEM. ANOVA was followed by the Tukey-Kramer test. Nx versus Sh mice: ${ }^{* *} P<0.01$. 
A

Poly I:C or placebo

$\Gamma$

B6

$\begin{array}{ccc}-7 & D\end{array}$
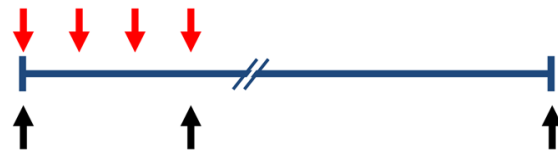

First Nx Second Nx

or Sh

or Sh

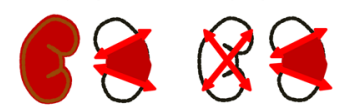

C

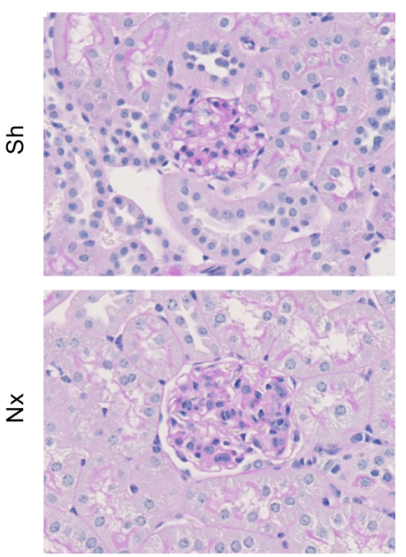

D
C Placebo

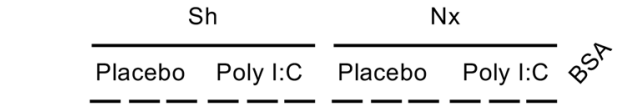

Poly I:C
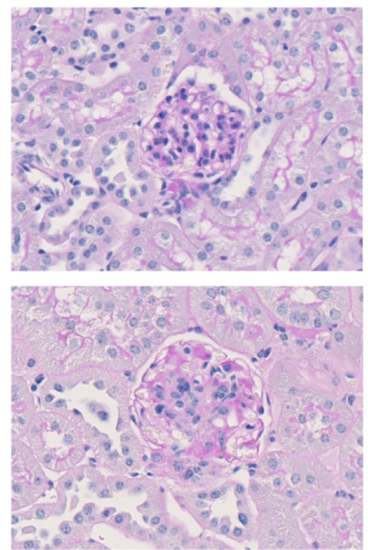

$72 \mathrm{kDa}$
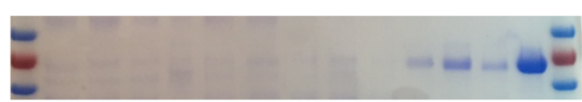

B

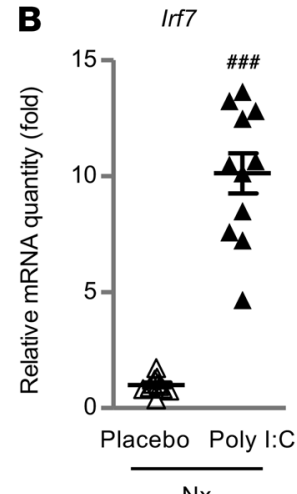

$\mathrm{Nx}$
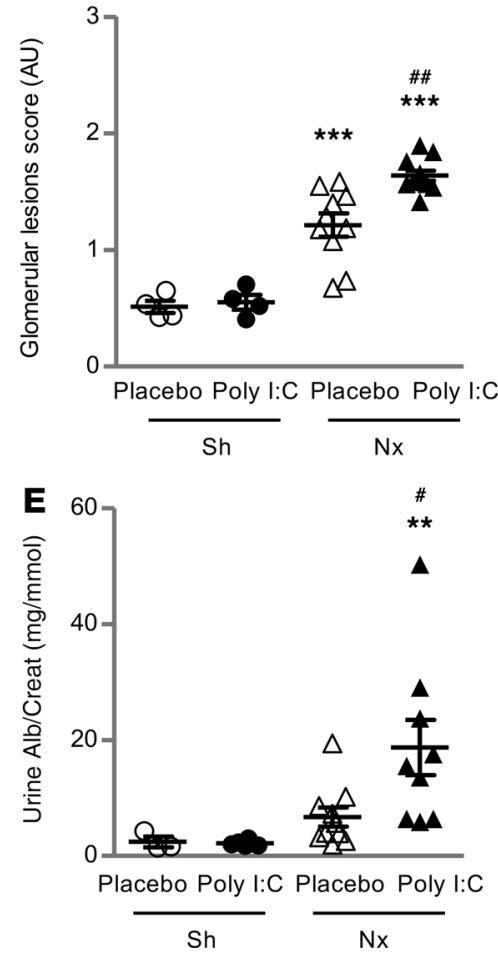

Figure 9. Early IFN activation induces glomerulosclerosis after nephron reduction in resistant B6 mice. (A) Experimental protocol used for IFN response induction in initially resistant B6 mice. Mice were injected intraperitoneally with poly(I:C) or placebo (saline buffer) every 2 days during the week after the first nephrectomy ( $\mathrm{Nx}, n=12$ per group) or sham operation (Sh, $n=6$ per group). The first Nx corresponded to the excision of the 2 poles of the left kidney. The contralateral (right) kidney (second $\mathrm{Nx}$ ) was removed 1 week after the first surgery and mice were sacrificed 16 weeks after the second $\mathrm{Nx}$. (B) Renal mRNA expression of Irf7 and SiglecH in placebo- and poly(I:C)-treated NxB6 mice at the time of the second Nx surgery ( $n=11$ per group). Data are shown

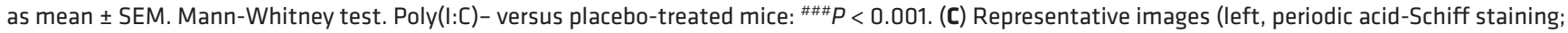
original magnification, $\times 400$ ) and quantification (right) of glomerular lesions from ShB6 and NxB6 mice injected with placebo or poly(I:C) for 1 week and sacrificed 16 weeks afterward ( $n=6$ and $10-11$ for Sh and Nx, respectively, in each group). Data are shown as mean \pm SEM. ANOVA was followed by the Tukey-Kramer test. Nx versus Sh mice: ${ }^{* *} P<0.001$. Poly(I:C)- versus placebo-treated mice: ${ }^{\# \# P} 0.01$. (D) Representative urine Coomassie staining and (E) albumin-to-creatinine ratio in ShB6 and NxB6 mice injected with placebo or poly(I:C) for 1 week and sacrificed 16 weeks afterward ( $n=6$ and $10-11$ for Sh and Nx, respectively, in each group). BSA served as the positive control. Data are shown as mean \pm SEM. ANOVA was followed by the Tukey-Kramer test. Nx versus Sh mice: ${ }^{* *} P<0.01$. Poly $(\mathrm{I}: \mathrm{C})$ - versus placebo-treated mice: ${ }^{\#} P<0.05$.

Protocols. Surgery was performed under xylazine (Rompun 2\%; Bayer, Leverkusen, France) (6 $\mu \mathrm{g} / \mathrm{g}$ of body weight) and ketamine (Clorketam 1000; Vetoquinol SA, Lirre, France) (120 $\mu \mathrm{g} / \mathrm{g}$ of body weight) anesthesia. Subtotal nephrectomy (Nx) was performed as previously described (40). Briefly, the 2 poles of the left kidney were excised to reach $75 \%$ reduction of total renal mass (first $\mathrm{Nx}$ ), and 1 week after, the right kidney (second $\mathrm{Nx}$ ) was removed on 48 mice of each strain (12 mice for each time point). Thirty mice of each strain (6 mice for each time point) were subjected to sham operation (Sh) and used as controls. After surgery, all mice were fed a defined diet containing $20 \%(\mathrm{w} / \mathrm{w})$ casein and $0.5 \%$ sodium $(41)$. Mice from each group (ShB6, NxB6, ShFVB, and NxFVB) were sacrificed at 2, 7, 28, and 56 days after 
surgery (second $\mathrm{Nx}$ ). At each time point, $4 \mathrm{Sh}$ and $6 \mathrm{Nx}$ mice were injected i.p. 4 hours before sacrifice with 5-ethynyl-2'-deoxyuridine (200 $\mu \mathrm{g} / \mathrm{g}$ of body weight, Invitrogen, Thermo Fisher Scientific, A10044) in $0.9 \%$ saline. At the time of sacrifice, kidneys were removed, weighed, and analyzed by morphological studies, immunohistochemistry, Western blot, and real-time PCR.

For functional studies, 1 week before sacrifice, blood pressure was recorded in both Sh $(n=3$ for each strain) and $\mathrm{Nx}$ ( $n=3$ for each strain) B6 and FVB mice for 3 consecutive days, using tail-cuff plethysmography and the BP-2000 system (Bioseb). Urine samples were also collected 24 hours before sacrifice ( $n=6$ and 9 for Sh and Nx mice, respectively, for each strain). Blood was collected by cava puncture at the time of sacrifice.

For poly(I:C) experiments, B6 mice were injected i.p. with $200 \mu \mathrm{g}$ poly(I:C) (InvivoGen) or placebo (saline buffer) every 2 days during the week after the first $\mathrm{Nx}$ ( $n=12$ per group) or Sh ( $n=6$ per group). The contralateral kidney removed at the time of second $\mathrm{Nx}$ was used as an internal control to determine the activation of IFN-stimulated genes. Urine was collected regularly to determine the presence of proteinuria until sacrifice 16 weeks after $\mathrm{Nx}$.

The UUO model was performed on 9-week-old B6 female mice. Briefly, the left ureter was exposed through a flank incision, and the ureter was ligated with 8-0 polydioxanone suture. The right kidney was used as an internal control. In parallel, sham operation was performed in control mice. Mice were sacrificed 7, 14, and 21 days after surgery. At the time of sacrifice, kidneys were removed, weighed, and analyzed by real-time PCR. At each time point, 10 UUO mice and 5 Sh mice were studied.

Gene profiling and microarray analysis. At 2, 28, and 56 days after surgery, 4 Sh and 4 Nx mice of each strain (FVB and B6) were used for whole-kidney transcriptomic analysis (Supplemental Figure 2). mRNA was extracted from nitrogen-frozen whole kidney using QIAzol Reagent and the miRNeasy Kit (QIAGEN) according to the manufacturer's instructions. Overall, the microarray analysis was performed on 48 mice using GeneChip Mouse Genome 4302.0 (Affymetrix, Thermo Fisher Scientific). Technical quality was checked by visual inspection of density, RNA degradation, and MA plots. All chips were of high technical quality with no irregular degradation pattern and were used for further analysis.

Renal function. Urinary protein, albumin, and creatinine concentrations were measured using an Olympus AU400 multiparametric analyzer (Instrumentation Laboratory). In addition, Coomassie gels were used to visualize albuminuria. Plasma creatinine levels were determined using a Konelab 20i analyzer (Thermo Fisher Scientific).

Morphological analysis. Kidneys were fixed in 4\% paraformaldehyde and paraffin-embedded, and 4- $\mu \mathrm{m}$ sections were stained with periodic acid-Schiff, Masson's trichrome, H\&E, and picrosirius red. Images were acquired using a Nikon Digital Camera Dx/m/1200. All sections were evaluated by a renal pathologist who was unaware of the group studied. The degree of acute tubular lesions at day 2 was evaluated using a semiquantitative injury score as previously described (42). The degree of glomerular lesions was evaluated at an original magnification of $\times 400$ on 40 to 60 glomeruli per mouse using the following scoring system: 0 , no lesion; 1 , mild sclerosis involving up to $25 \%$ of the glomerulus; 2 , moderate sclerosis involving $25 \%$ to $50 \%$ of the glomerulus; 3 , severe sclerosis involving $50 \%$ to $75 \%$ of the glomerulus; and 4 , global sclerosis involving more than $75 \%$ of the glomerulus. The degree of tubular dilations was automatically quantified using NIS Elements AR software, version 3.00 (Laboratory Imaging Ltd.), as previously described (18). The extent of interstitial fibrosis was also quantified using NIS Elements AR software. Staining of all kidney samples with picrosirius was performed simultaneously, and red intensity above a defined threshold was defined as fibrosis. At least 10 random selected fields (original magnification, $\times 200$ ) across the corticomedullary junction were analyzed per each kidney section, and the results were expressed as percentages of the total area of the selected fields.

Immunohistochemistry. Four-micrometer sections of paraffin-embedded kidneys were submitted to heat-mediated antigen retrieval if required and incubated with primary antibodies against Ki-67 (1:100, ab16667, Abcam, Inc.), CD3 (1:100; ab16669; Abcam, Inc.), F4/80 (1:100; MCA497; AbD Serotec), Ly6B (1:100, ab53457, Abcam, Inc.), phospho-histone H3 (Ser10) (1:100; 9716, Cell Signaling Technology), p-H2A.X (Ser139) (1:100; 2577, Cell Signaling Technology), p21 (1:250, MilliporeSigma, clone OP76n, Calbiochem), and KIM1 (1:100, AF1817, R\&D Systems, Bio-Techne), followed by the appropriate horseradish peroxidase-conjugated secondary antibodies.

For colocalization experiments, proliferative cells were detected using anti-mouse Ki-67 (1:100, ab16667, Abcam, Inc.) and specific tubular markers: fluorescein-labeled LTL (1:250; FL-1321, Vector Labs) for the proximal tubules, antibody against TH $(1: 250 ; 8595-0054$, Bio-Rad) for the thick ascending Henle 
loop and distal tubules, and biotinylated DBA (1:250; B10-35, Vector Labs) for the collecting ducts. Images were acquired using a Leica SP8 confocal microscope (Leica Microsystems) and the Leica imaging software.

For PCNA and $\alpha$-SMA colocalization experiments, sections were first incubated with a mouse anti-PCNA antibody $\left(1: 100, \mathrm{M} 0879\right.$, Dako) overnight at $4^{\circ} \mathrm{C}$. Thereafter, sections were incubated with a secondary anti-mouse alkaline phosphatase-conjugated antibody (1:50, A1682, MilliporeSigma). Alkaline phosphatase activity was revealed using NBT/BCIP solution (11681451001, Roche). Sections were then fixed in $4 \%$ PFA for 15 minutes at $37^{\circ} \mathrm{C}$ and then incubated with a mouse anti- $\alpha$-SMA antibody (1:2000, A-5228, MilliporeSigma) overnight at $4^{\circ} \mathrm{C}$. Thereafter, sections were incubated with a secondary antimouse horseradish peroxidase-conjugated antibody (1:200, A16084, Invitrogen, Thermo Fisher Scientific) for 30 minutes at room temperature. Peroxidase activity was revealed by DAB staining (K3468, Dako).

The overall tubular proliferation index was calculated as the number of $\mathrm{Ki}-67^{+}$nuclei for the total number of tubular nuclei in at least 10 random fields per kidney section, as previously described (18). The tubular proliferation index per nephron segment was calculated as the number of Ki- $67^{+}$nuclei for the total number of tubular nuclei in a specific nephron segment $\left(\mathrm{LTL}^{+}, \mathrm{TH}^{+}\right.$, or $\left.\mathrm{DBA}^{+}\right)$. All tubular sections of each field were scored in at least 10 randomly selected fields per mice.

For p21 quantification, the number of $\mathrm{p} 21^{+}$tubular nuclei was counted in at least 10 random fields per kidney section (original magnification, $\times 200$ ), and the results were expressed as the average value per section.

For KIM1 quantification, the number of $\mathrm{KIM}^{+}$tubular sections was counted in at least 10 random fields per kidney section (original magnification, $\times 200$ ), and the results were expressed as the average value per each section.

For quantification of $\mathrm{CD}^{+}$and $\mathrm{F} 4 / 80^{+}$renal cells, the degrees of cellular infiltrate were automatically quantified using ImageJ software $(\mathrm{NIH})$ and expressed as the percentage of positive staining per field. At least 10 fields per section were examined at the original magnification of $\times 200$.

Detection of 5-ethynyl-2'-deoxyuridine and cell cycle progression studies. 5-Ethynyl-2'-deoxyuridine (EdU) labeling and coimmunostaining with anti-Ki-67 and anti-phospho-histone H3 (Ser10) antibodies were performed according to the manufacturer's instructions. Briefly, after paraffin removal, sections were submitted to heat antigen retrieval and incubated with primary antibody to anti-mouse Ki-67 (1:100, ab16667, Abcam, Inc.). EdU detection was then performed according to the manufacturer's instructions using the Click-iT EdU Alexa Fluor 488 imaging kit (Invitrogen, Thermo Fisher Scientific). Sections were finally counterstained with Hoechst and mounted for fluorescence microscopy using a Leica SP8 confocal microscope (Leica Microsystems) and the Leica imaging software. Quantification analysis was performed using ImageJ software on at least 10 randomly selected microscopic fields from the cortex and corticomedullary junction in $4 \mathrm{Nx}$ mice from each strain and at each time point. The overall number of tubular epithelial proliferating cells was determined using Ki-67 staining.

EdU incorporation and phospho-histone H3 (Ser10) staining were used to identify the number of cells in $S$ and $G_{2} / M$ phases, respectively. Subsequently, the number of tubular epithelial cells in $G_{1}$ phase was calculated by subtracting the number of cells in $\mathrm{S}$ phase $\left(\mathrm{EdU}^{+}\right)$and $\mathrm{G}_{2} / \mathrm{M}$ phase (phospho-histone $\mathrm{H}^{+}$) from the total number of proliferating cells $\left(\mathrm{Ki}-67^{+}\right)$. The percentage of cells in each phase was expressed relative to all $\mathrm{Ki}-67^{+}$tubular epithelial cells.

TUNEL assay. Apoptosis was detected in $4-\mu \mathrm{m}$ sections of paraffin-embedded kidneys by TUNEL assay using the In Situ Cell Death Detection Kit (Roche) according to the manufacturer's protocol. All the microscopic fields of a whole kidney section were quantified, and results are presented as the number of $\mathrm{TUNEL}^{+}$tubular cells per field.

Real-time RT-PCR. For mRNA analysis, reverse transcription was performed using the High-Capacity Reverse-Transcription Kit (Life Technologies). Quantitative real-time PCR was performed using the SYBR Green method and CFX96 Touch Real-Time PCR Detection System (Bio-Rad). Primers (Eurogentec) are provided in Supplemental Table 1. Levels of expression were determined by normalization to the housekeeping gene Rpl13.

Western blot analysis. Western blot analyses were performed as previously described $(8,18)$. Briefly, protein extracts from kidneys were resolved by SDS-PAGE before being transferred onto the appropriate membrane and incubated with primary antibodies against phospho-histone H2A.X (Ser139) (1:100; 2577, Cell Signaling Technology) and GAPDH (1:10000; Mab374, MilliporeSigma) followed by the appropriate Alexa Fluor-conjugated secondary antibody at 1:5000 (Life Technologies). Fluorescence was acquired using a ChemiDoc MP Imaging System (Bio-Rad), and densitometry was performed using Image Lab software 5.0. 
Microarray analysis. Raw data were normalized with the MAS5 method using the affy software package in $\mathrm{R} /$ Bioconductor and consequently $\log _{2}$-transformed. Chips were controlled for quality by an in-house pipeline that detects both technical and biological issues (43). Data were deposited in the National Center for Biotechnology Information's Gene Expression Omnibus database (GSE148084). Between-sample normalization was performed using the quantile method implemented in the limma package. Unbiased clustering analysis was performed using coinertia analysis implemented in the made4 package. Differential gene expression was detected by a linear model that considers strain (FVB or B6), surgery (Sh or Nx), time (day 2, 28, or 56), and all interactions among the 3 factors using limma. The quality of the model was checked and confirmed by visual inspection of $P$ value histograms and volcano plots. Gene set enrichment analysis was performed using GSEA with 1000 permutations (44). A collection of gene signatures from Pathway Interaction Database, Pathway Commons, and Reactome was used. GSEA results are reported in enrichment scores defined as ES $=$ sign(NES) $\left|\log _{10}(\mathrm{FDR})\right|$, i.e., the sign of normalized enrichment score (NES) reported by GSEA ( 1 if NES > 0, 0 if NES $=0$, and -1 if NES $<0$ ) multiplied by the absolute $\log _{10}$ transformation of the FDR reported by GSEA. Enrichment scores range between -4 and 4 , corresponding to cases where NES is negative or positive and $P=0.0001$.

Statistics. Data are expressed as means \pm SEMs. Differences between the experimental groups were evaluated using 1-way ANOVA followed, when significant $(P<0.05)$, by the Tukey-Kramer test. When only 2 groups were compared, Mann-Whitney $U$ test was used. A $P$ value of less than 0.05 was considered significant. The statistical analysis was performed using GraphPad Prism software.

Study approval. Animal procedures were approved by the departmental director of "Services Vétérinaires de la Préfecture de Police de Paris" and by the ethical committee of the Paris Descartes University.

\section{Author contributions}

MZ designed and performed the experiments, analyzed data, and wrote the paper. PB, FV, S Garbay, LY, and S Germano also performed some experiments and analyzed data. $\mathrm{MB}, \mathrm{TB}$, and $\mathrm{CN}$ performed mouse experiments (breeding, surgery, and treatments). JDZ and LB performed transcriptomic analysis. MCG analyzed the morphology. MG and GF revised the manuscript. FT and MP provided the conceptual framework, designed the study, supervised the project, and wrote the paper.

\section{Acknowledgments}

We thank Sophie Berissi, Noémie Gadessaud, Christine Bole, and the LEAT, Histology, and Genomics Platforms for technical assistance. This work was supported by INSERM, Université Paris Descartes, AP-HP, Agence Nationale Recherche, Whoami Laboratoire d'Excellence, Pharma Research and Early Development Roche Laboratories (Basel, Switzerland), and Institut Roche de Recherche et Médecine Translationnelle (Paris, France).

Address correspondence to: Fabiola Terzi, INSERM U1151, CNRS UMR8253, "Mechanisms and Therapeutic Strategies of Chronic Kidney Disease,” Faculté de Médecine Necker, Université de Paris, 156-160 Rue de Vaugirard, 75015 Paris, France. Phone: 33.1.40615364; Email: fabiola.terzi@inserm.fr.

1. Levin A, et al. Global kidney health 2017 and beyond: a roadmap for closing gaps in care, research, and policy. Lancet. 2017;390(10105):1888-1917.

2. Webster AC, Nagler EV, Morton RL, Masson P. Chronic kidney disease. Lancet. 2017;389(10075):1238-1252.

3. Remuzzi G, Benigni A, Remuzzi A. Mechanisms of progression and regression of renal lesions of chronic nephropathies and diabetes. J Clin Invest. 2006;116(2):288-296.

4. Hostetter TH, Olson JL, Rennke HG, Venkatachalam MA, Brenner BM. Hyperfiltration in remnant nephrons: a potentially adverse response to renal ablation. Am J Physiol. 1981;241(1):F85-F93.

5. Hostetter TH. Progression of renal disease and renal hypertrophy. Annu Rev Physiol. 1995;57:263-278

6. Kren S, Hostetter TH. The course of the remnant kidney model in mice. Kidney Int. 1999;56(1):333-337.

7. Terzi F, et al. Targeted expression of a dominant-negative EGF-R in the kidney reduces tubulo-interstitial lesions after renal injury. J Clin Invest. 2000;106(2):225-234.

8. Sun ZY, et al. High-resolution aliphatic side-chain assignments in $3 \mathrm{D}$ HCcoNH experiments with joint $\mathrm{H}-\mathrm{C}$ evolution and non-uniform sampling. J Biomol NMR. 2005;32(1):55-60.

9. Pillebout E, et al. Proliferation and remodeling of the peritubular microcirculation after nephron reduction: association with the progression of renal lesions. Am J Pathol. 2001;159(2):547-560.

10. Pillebout E, et al. JunD protects against chronic kidney disease by regulating paracrine mitogens. J Clin Invest. 2003;112(6):843-852.

11. Esposito C, He CJ, Striker GE, Zalups RK, Striker LJ. Nature and severity of the glomerular response to nephron reduction is 
strain-dependent in mice. Am J Pathol. 1999;154(3):891-897.

12. Ma LJ, Fogo AB. Model of robust induction of glomerulosclerosis in mice: importance of genetic background. Kidney Int 2003;64(1):350-355.

13. Laouari D, et al. TGF-alpha mediates genetic susceptibility to chronic kidney disease. J Am Soc Nephrol. 2011;22(2):327-335

14. Laouari D, et al. A transcriptional network underlies susceptibility to kidney disease progression. EMBO Mol Med. 2012;4(8):825-839.

15. Yang L, Besschetnova TY, Brooks CR, Shah JV, Bonventre JV. Epithelial cell cycle arrest in G2/M mediates kidney fibrosis after injury. Nat Med. 2010;16(5):535-543.

16. Krämer A, Green G, Pollard J Jr, Tugendreich S. Causal analysis approaches in Ingenuity Pathway Analysis. Bioinformatics. 2014;30(4):523-530

17. Vogel H, et al. Loss of function of Ifi202b by a microdeletion on chromosome 1 of C57BL/6J mice suppresses $11 \beta$-hydroxysteroid dehydrogenase type 1 expression and development of obesity. Hum Mol Genet. 2012;21(17):3845-3857.

18. Viau A, et al. Lipocalin 2 is essential for chronic kidney disease progression in mice and humans. J Clin Invest. 2010;120(11):4065-4076.

19. Kang HM, et al. Defective fatty acid oxidation in renal tubular epithelial cells has a key role in kidney fibrosis development. Nat Med. 2015;21(1):37-46.

20. Megyesi J, Price PM, Tamayo E, Safirstein RL. The lack of a functional p21(WAF1/CIP1) gene ameliorates progression to chronic renal failure. Proc Natl Acad Sci U S A. 1999;96(19):10830-10835.

21. Lovisa S, et al. Epithelial-to-mesenchymal transition induces cell cycle arrest and parenchymal damage in renal fibrosis. Nat Med. 2015;21(9):998-1009.

22. Sadler AJ, Williams BR. Interferon-inducible antiviral effectors. Nat Rev Immunol. 2008;8(7):559-568

23. Theofilopoulos AN, Baccala R, Beutler B, Kono DH. Type I interferons (alpha/beta) in immunity and autoimmunity. Annu Rev Immunol. 2005;23:307-336.

24. Anders HJ, Lichtnekert J, Allam R. Interferon-alpha and -beta in kidney inflammation. Kidney Int. 2010;77(10):848-854

25. Fairhurst AM, et al. Type I interferons produced by resident renal cells may promote end-organ disease in autoantibody-mediated glomerulonephritis. J Immunol. 2009;183(10):6831-6838.

26. Baccala R, Gonzalez-Quintial R, Schreiber RD, Lawson BR, Kono DH, Theofilopoulos AN. Anti-IFN- $\alpha / \beta$ receptor antibody treatment ameliorates disease in lupus-predisposed mice. J Immunol. 2012;189(12):5976-5984.

27. Migliorini A, et al. The antiviral cytokines IFN- $\alpha$ and IFN- $\beta$ modulate parietal epithelial cells and promote podocyte loss: implications for IFN toxicity, viral glomerulonephritis, and glomerular regeneration. Am J Pathol. 2013;183(2):431-440.

28. Aghajan M, et al. Antisense oligonucleotide treatment ameliorates IFN- $\gamma$-induced proteinuria in APOL1-transgenic mice. JCI Insight. 2019;4(12):126124.

29. Gresser I, Maury C, Tovey M, Morel-Maroger L, Pontillon F. Progressive glomerulonephritis in mice treated with interferon preparations at birth. Nature. 1976;263(5576):420-422.

30. Trinchieri G. Type I interferon: friend or foe? J Exp Med. 2010;207(10):2053-2063.

31. Kumar H, Kawai T, Akira S. Pathogen recognition by the innate immune system. Int Rev Immunol. 2011;30(1):16-34.

32. Paludan SR, Bowie AG. Immune sensing of DNA. Immunity. 2013;38(5):870-880.

33. Härtlova A, et al. DNA damage primes the type I interferon system via the cytosolic DNA sensor STING to promote anti-microbial innate immunity. Immunity. 2015;42(2):332-343.

34. Yu Q, et al. DNA-damage-induced type I interferon promotes senescence and inhibits stem cell function. Cell Rep. 2015;11(5):785-797.

35. Yang L, et al. KIM-1-mediated phagocytosis reduces acute injury to the kidney. J Clin Invest. 2015;125(4):1620-1636.

36. Shang D, Yang P, Liu Y, Song J, Zhang F, Tian Y. Interferon- $\alpha$ induces G1 cell-cycle arrest in renal cell carcinoma cells via activation of Jak-Stat signaling. Cancer Invest. 2011;29(5):347-352.

37. Yano $\mathrm{M}$, et al. Hepatitis B virus $\mathrm{X}$ induces cell proliferation in the hepatocarcinogenesis via up-regulation of cytoplasmic $\mathrm{p} 21$ expression. Liver Int. 2013;33(8):1218-1229.

38. Ohkoshi S, Yano M, Matsuda Y. Oncogenic role of p21 in hepatocarcinogenesis suggests a new treatment strategy. World J Gastroenterol. 2015;21(42):12150-12156.

39. Sangfelt O, et al. Molecular mechanisms underlying interferon-alpha-induced G0/G1 arrest: CKI-mediated regulation of G1 Cdk-complexes and activation of pocket proteins. Oncogene. 1999;18(18):2798-2810.

40. Terzi F, et al. Reduction of renal mass is lethal in mice lacking vimentin. Role of endothelin-nitric oxide imbalance. JClin Invest. 1997;100(6):1520-1528

41. Terzi F, Beaufils H, Laouari D, Burtin M, Kleinknecht C. Renal effect of anti-hypertensive drugs depends on sodium diet in the excision remnant kidney model. Kidney Int. 1992;42(2):354-363.

42. Wu H, et al. TLR4 activation mediates kidney ischemia/reperfusion injury. J Clin Invest. 2007;117(10):2847-2859.

43. Zhang JD, et al. Detect tissue heterogeneity in gene expression data with BioQC. BMC Genomics. 2017;18(1):277.

44. Subramanian A, et al. Gene set enrichment analysis: a knowledge-based approach for interpreting genome-wide expression profiles. Proc Natl Acad Sci U S A. 2005;102(43):15545-15550. 